\title{
Shared Relay Assignment (SRA) for Many-to-One Traffic in Cooperative Networks
}

\author{
Hongli Xu, Member, IEEE, Liusheng Huang, Senior Member, IEEE, Chunming Qiao, Fellow, IEEE, \\ Xinglong Wang, Shan Lin, Member, IEEE, and Yu-e Sun,
}

\begin{abstract}
Relay assignment significantly affects the performance of the cooperative communication, which is an emerging technology for the future mobile system. Previous studies in this area have mostly focused on assigning a dedicated relay to each sourcedestination pair for one-to-one (121) traffic. However, many-to-one (M21) traffic, which is also common in many situations (for example, several users associate with one access point in a wireless access network such as a WLAN), hasn't been well studied. This paper addresses the shared relay assignment (SRA) problem for M21 traffic. We formulate two new optimization problems: one is to maximize the minimum throughput among all the sources (hereafter called M21-SRA-MMT), and the other is to maximize the total throughput over all the sources while maintaining some degree of fairness (hereafter called M21-SRA-MTT). As the optimal solutions tp the two problems are hard to find, we propose two approximation algorithms whose performance factors are 5.828 and 3, respectively, based on the rounding mechanism. Extensive simulation results show that our algorithms for M21-SRA-MMT can significantly improve the minimum throughput compared with existing algorithms, while our algorithm for M21-SRA-MTT can achieve the close-to-optimal performance.
\end{abstract}

Index Terms-Cooperative Communication, Shared Relay Assignment, Max-Min Throughput, Max-Total Throughput, Fairness.

\section{INTRODUCTION}

$\mathrm{R}$ ECENT studies on cooperative communication (CC) [1][7] show its potential to cope with lossy wireless channels using spatial diversity. Moreover, spatial diversity can be achieved by exploiting the antennas on other devices, called relay nodes, in the network under the cooperative communication scheme. Thus, the cooperative communication becomes one of the emerging technologies for the next-generation mobile systems [8]-[10]. In scenarios where a source (e.g., a mobile phone) and a destination (e.g., the base station) communicate with each other in two hops or less, there are two main cooperative modes, namely amplify-and-forward $(A F)$ and decode-and-forward $(D F)$, respectively [3].

The previous studies in [11]-[13] have concluded that the choice of relay nodes played a significant role in achieving transmission performance (e.g., capacity and throughput) under both cooperative modes. Choosing an appropriate assignment of relay nodes will provide a higher capacity for the source-destination (or $s$ - $d$ for short) pair than using direct transmission (DT). In particular, Zhao et al. [11] showed that it was sufficient to select the best relay node for a given $s$ - $d$

- H. Xu, L. Huang, X. Wang are with the School of Computer Science and Technology, University of Science and Technology of China, Hefei, Anhui, 230027; and Suzhou Institute for Advanced Study, University. of Science and Technology of China, Suzhou, Jiangsu 215123. E-mail: \{xuhongli, lshuang\}@ustc.edu.cn,wxlong@mail.ustc.edu.cn.

- C. Qiao is with the Department of Computer Science and Engineering, State University of NY, Buffalo NY 14260-2000, USA. E-mail: qiao@computer.org.

- S. Lin is with the Department of Computer and Information Sciences, Temple University, Philadelphia, PA 19122. E-mail: slin@temple.edu.

- Y. Sun is with the School of Urban Rail Transportation, Soochow University, Suzhou, Jiangsu, 215006. E-mail: sunye12@suda.edu.cn. pair to achieve the full diversity, and thus there was no need to have multiple relay nodes for the cooperative transmission of a $s-d$ pair (which in fact would also need more complex network management and precise time synchronization among several relay nodes [1]). Accordingly, the relay assignment problem has been widely studied in recent years, mostly under the de facto assumption that at most one relay node will be used for a given $s$ - $d$ pair.

In the mobile access network such as a WLAN, each user expects to obtain a maximum bandwidth or throughput so as to improve the QoS of different applications. As the cooperative communication can improve the spatial diversity in the wireless network, this will help to increase the transmission throughput for each user in the WLAN. Now, we consider a common situation under the WLAN scene. For example, in a managed WLAN, there has a few access points (APs), and also puts in some relay nodes. Several terminal users (e.g., laptops or WIFI-compatible devices) will connect with one same AP using the previous association control method [14] for Internet access, which belongs to many-to-one (M21) traffic. In this application, the system will run a centralized management algorithm to select/assign relays to the users so as to reach the different QoS performances, such as max-min throughput or max-total throughput, etc. Therefore, it is of great importance to implement the relay assignment for M21 traffic in cooperative wireless networks.

Existing research in $\mathrm{CC}$ has focused only on one-to-one (121) traffic, but not on many-to-one traffic. As an example, the authors in [1] [15] studied relay assignment where multiple independent $s-d$ pairs competed for a set of relay nodes. In particular, they assumed that a relay node could be used by only one $s$-d pair - a policy which we would refer to as dedicated relay assignment or DRA. As shown in Fig.1(a), 


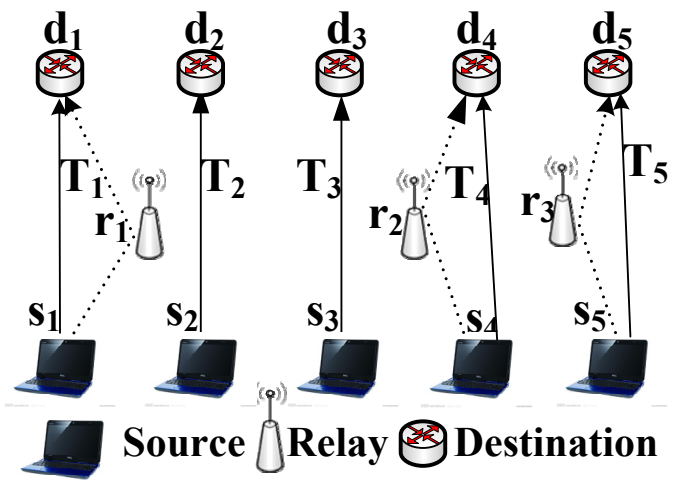

(a) DRA in One-to-One (121)

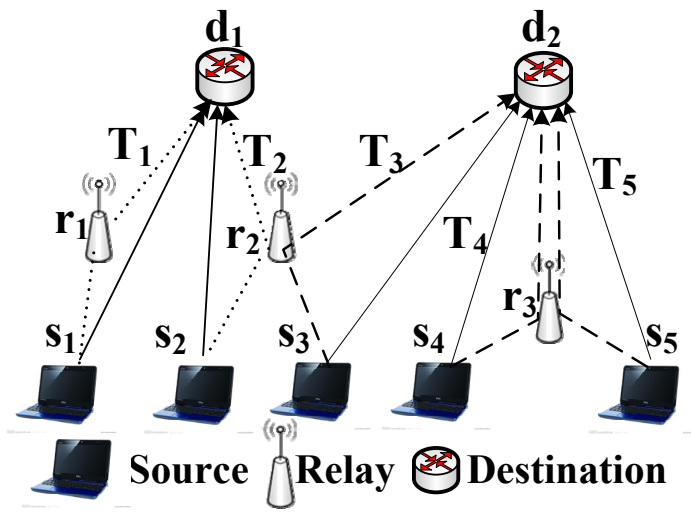

(b) SRA in Many-to-One (M21)

Fig. 1: Different Relay Assignments and Traffic Patterns

the relay node $r_{1}$ is only used by the source node $s_{1}$, and $r_{2}$ is by $s_{4}$. Under such an assumption, they proposed an optimal algorithm, called ORA, based on linear marking to maximize the minimum throughput (MMT) among all the sources in a network. We will refer to this problem as 121-DRA-MMT. As another example, Yang et al. [16] studied the problem of relay assignment for multiple $s$ - $d$ pairs to maximize the total throughput (MTT) over all the sources in a network. Although their work considered the possibility of sharing a relay node by multiple $s-d$ pairs, they proved that each relay node should be assigned to only one $s$ - $d$ pair so as to achieve the MTT objective. In other words, the DRA policy should also be enforced for MTT. Additionally, they used maximum weighted bipartite matching to solve the problem in polynomial time. We will refer to this problem as 121-DRA-MTT.

In this paper, we study several new relay assignment problems for M21 traffic as shown in Fig.1(b), under the same default assumption of at most one relay node per $s$ - $d$ transmission pair. These new problems also use different relay assignment policies including shared relay assignment (SRA) with which, the same relay can (but does not have to) be used by more than one source. For example, source nodes $s_{2}$ and $s_{3}$ will share the relay node $r_{2}$ to cooperatively communicate with the destination nodes $d_{1}$ and $d_{2}$, respectively. More specifically, we focus on two new problems: M21-SRA-MMT and M21-SRA-MTT.

Note that, firstly, in several practical wireless applications, multiple source nodes may associate with one common destination node (which serves as a sink or access point), so it is meaningful to study relay assignment for M21 traffic. Secondly, the case with M21 traffic and the SRA policy is more general than that with 121 traffic and DRA, since the latter can be considered as a special case of the former where there is (at most) one relay per source and only one source per destination. In fact, our solutions described in this work for the former cases will be applicable to the cases with strictly M21 traffic, strictly 121 traffic or a mixed M21 and 121 traffic. Thirdly, considering M21 traffic and the SRA policy leads to open problems and new challenges yet to be addressed in order to take the full advantage of SRA. For example, in 121-DRAMTT [16], the relay assignment problem can be transformed into the bipartite matching problem where the bipartite graph consists of the set of sources on one side and the set of relay nodes on the other side. However, bipartite matching is no longer applicable when two or more sources actually share a relay node. In particular, when multiple sources share the same relay node, one has to consider the constraint that each of these sources will use the shared relay node for only a fraction of the time.

Moreover, when multiple sources send to the same destination as in M21 traffic, we need to consider the constraint that each of these sources can send to the common destination for only a fraction of the time. In fact, it is due to the destination access time constraint that makes our problems M21-SRAMMT and M21-SRA-MTT different from the previous ones.

Due to the destination access time constraint in M21 traffic, when pursuing the MMT objective as in [1] [15], allowing SRA would provide more flexibility in choosing a better relay for each source. This is because no sources (sharing a destination with some other sources) can transmit $100 \%$ of the time anyway, and hence dedicating a relay node to such a source as in the early works [1] [15] could be wasteful. In other words, SRA is expected to be able to achieve a higher minimum throughout, which also implies better maxmin fairness. Nevertheless, new and efficient relay assignment algorithms are needed to solve the M21-SRA-MMT problem.

More specifically, due to the destination access time constraint imposed on all the sources sending to the same destination, in order to pursue the MTT objective as in [16], one must consider the "fairness" issue. A reasonable fairness requirement is that the common destination will devote an equal amount of access time to each of its $k$ sources. In other words, each source will be able to transmit $\frac{1}{k}$ of the time to the common destination (using the CC or DT). Without any fairness requirement such as this, only one of the $k$ sources needs to be chosen per destination, such that the source can produce the maximum throughout at the common destination (with or without a relay), thus degenerating the solution to that of 121-DRA-MTT in [16], and resulting in starvation of all other sources sending to their common destination. However, with the fair (i.e., equal) destination access time requirement in place, it is now meaningful to study the SRA scheme, in conjunction with M21 traffic and the MTT objective. This clearly calls for new and efficient solutions to M21-SRA-MTT.

The main contributions of this work include: 
1) We formulate two new optimization problems, namely M21-SRA-MMT and M21-SRA-MTT, repsectively. To the best of our knowledge, this is the first time that SRA is considered in the context of M21 traffic.

2) For M21-SRA-MMT, we propose an approximation algorithm (RA-MM), which mainly uses the rounding mechanism to obtain the max-min throughput. This paper proves that the RA-MM algorithm can reach the approximation performance of $3+2 \sqrt{2}$ or about 5.828 .

3) We also present a variation of RA-MM to improve the average-case performance. The improved version, called IRA-MM, still guarantees the worst-case performance (i.e., the approximate factor of 5.828).

4) For M21-SRA-MTT under the equal destination access time constraint, a 3-approximation algorithm called RAMT is proposed.

5) Our simulation results show a high efficiency of the proposed algorithms. For example, our IRA-MM increases the minimum throughputs by about $66 \%$ when averaged over many cases, and up to $116 \%$ in some cases, compared with ORA [1], while the RA-MT algorithm can achieve close-to-optimal result for M21-SRA-MTT.

The rest of the paper is organized as follows. Section 2 gives the cooperative communication model, and the two problem definitions. Section 3 proposes two approximation algorithms for the M21-SRA-MMT problem. Section 4 addresses the M21-SRA-MTT problem. Simulation results are presented in Section 5. Related works are discussed in Section 6. We conclude the paper in Section 7.

\section{Preliminaries}

This section first reviews the mathematical expressions of transmission capacities under the CC and DT schemes. We then provide the problem definitions for M21-SRA-MMT and M21-SRA-MTT, respectively.

\subsection{Cooperative Communication Model}

For ease of expression, the variable $S N R_{s d}$ is used to denote the signal-noise-ratio from node $s$ to node $d$. There are two main forwarding modes for the cooperative communication, $A F$ and $D F$. Under the $A F$ mode, a cooperative relay node just amplifies the received signal from the source node and directly forwards it to the destination node without decoding the message. It has been shown in [3] that the capacity from node $s$ to node $d$ with a relay node $r$ is:

$$
C_{A F}(s, r, d)=W \cdot I_{A F}\left(S N R_{s d}, S N R_{s r}, S N R_{r d}\right)
$$

where $I_{A F}$ is the transmission gain under the $A F$ mode, with $I_{A F}=\frac{1}{2} \log _{2}\left(1+S N R_{s d}+\frac{S N R_{s r} \cdot S N R_{r d}}{S N R_{s r}+S N R_{r d}+1}\right)$, and $W$ is the bandwidth. Under the $D F$ mode, the relay node decodes the received signal, and re-encodes it before forwarding to the destination node. The capacity for the $D F$ mode is given as follows [3]:

$$
C_{D F}(s, r, d)=W \cdot I_{D F}\left(S N R_{s d}, S N R_{s r}, S N R_{r d}\right)
$$

where $I_{D F}$ is the transmission gain under the $D F$ scheme, and $I_{D F}=\frac{1}{2} \min \left\{\log _{2}\left(1+S N R_{s r}\right), \log _{2}\left(1+S N R_{s d}+S N R_{r d}\right)\right.$.

When the cooperative communication is not used, sender node $s$ transmits directly to receiver node $d$ in both time slots.
This is just the traditional communication scheme. Thus, the maximum rate achievable from node $s$ to node $d$ is:

$$
C_{D}(s, d)=W \cdot \log _{2}\left(1+S N R_{s d}\right)
$$

From the above theoretical results, although $A F$ and $D F$ are different cooperative modes, the expressions of their capacities are both the function of variables $S N R_{s d}, S N R_{s r}$ and $S N R_{r d}$ [1]. Then, the relay assignment algorithm designed for the $A F$ mode can be easily extended to $D F$, and vice versa. Thus, the relay node assignment algorithms developed in this paper can be applied to both the $A F$ and $D F$ modes.

\subsection{Problem Definition}

We consider three disjoint node sets in a network. One is the source node set, $S=\left\{s_{1}, s_{2}, \ldots, s_{n_{s}}\right\}$, with $n_{s}=|S|$; the second is the destination node set, $D=\left\{d_{1}, d_{2}, \ldots, d_{n_{d}}\right\}$, with $n_{d}=|D|$; Another is the relay node set, $R=\left\{r_{1}, r_{2}, \ldots, r_{n_{r}}\right\}$, with $n_{r}=|R|$. In many practical application scenarios, such as a large network with multiple cells or clusters, multiple source nodes $s_{i}$ (e.g., clients) may associate with one common destination node $d_{i^{\prime}}$ (e.g., the access point or sink node), resulting in what we call M21 traffic. We assume that each source node $s_{i}$ has established a connection with a destination node $d_{i^{\prime}}$ as in e.g., [14], etc, and will focus on SRA in such M21 traffic. For simplicity, the transmission from source $s_{i}$ to destination $d_{i^{\prime}}$ is denoted by $\Gamma_{i}=\left(s_{i}, d_{i^{\prime}}\right)$, with $1 \leq i \leq n_{s}$ and $1 \leq i^{\prime} \leq n_{d}$. In our many-to-one scheme, since multiple sources will not communicate with the same destination at exactly the same time, these sources and the associated destination can be assigned only one channel for cooperative communications. Thus, the number of required orthogonal channels is mainly determined by the deployment of destination nodes in a wireless network. Under many practical scenarios, the number of destination nodes (e.g., sinks or APs) is usually less than that of source nodes (e.g., terminals). Moreover, the destination nodes will not densely deployed. Hence, there should be enough such orthogonal channels available given that the same assumption holds in the multiple 121 schemes (i.e., $s$ - $d$ pairs). In order to avoid the transmission interference, we assume that orthogonal channels are available in the network (e.g., using OFDMA), which is also used for the cooperative communication [1] [15].

Zhao et al. [11] had shown that for a single-hop transmission, the diversity gain obtained by exploiting multiple relay nodes was not higher than that by selecting the best relay. Accordingly, it is reasonable to assume that at most one relay node will participate into cooperative communication for a certain transmission pair $\Gamma_{i}$. For uniform description, we add a virtual relay $r_{0}$, and denote $R^{+}=R \cup\left\{r_{0}\right\}$. When we say that source node $s_{i}$ uses the direct transmission, it is equivalent to say that this source node selects the virtual relay $r_{0}$.

As multiple transmission pairs may share a relay node and destination node, each source node $s_{i}$ will be allocated a transmission time $t_{i}$, such that the time constraint on each relay node as well as each destination node will be satisfied. Once the relay assignment and transmission time are determined, each source node $s_{i}$ can achieve a certain throughput $B_{i}$. Our objectives are to maximize the minimum throughput and 
the total throughput, respectively. We give the formal problem definition as follows.

Problem Definition: Consider a network where each source node $s_{i} \in S$ has associated with a destination node $d_{i^{\prime}} \in D$, and multiple source nodes may associate with one common destination node. We assume that each source node will select at most one relay node for cooperative transmission, and such a relay node may be shared by multiple source nodes, which may or may not have the same destination (see Fig.1(b) for illustration). Moreover, each source node $s_{i}$ will be allocated a transmission time $t_{i}$, so that the total transmission times of each relay node and each destination node do not exceed one unit. Then, each source node $s_{i}$ will obtain the achievable throughput $B_{i}$. Two problems are defined with different optimization objectives. The first problem, called M21-SRAMMT, aims to maximize the minimum throughput of all the source nodes, which is expressed by:

$$
\operatorname{Max}\left\{\min B_{i}, s_{i} \in S\right\}
$$

The other problem, called M21-SRA-MTT, will optimize the max-total throughput in the network. That is,

$$
\operatorname{Max} \sum_{s_{i} \in S} B_{i}
$$

Note that, if the transmission pair and relay node are regarded as the job and processor, M21-SRA-MMT is similar to the unrelated processor scheduling (UPS) problem [17]. However, the main difference is that there is time constraint on the transmission pairs (i.e., destination nodes) in the M21SRA-MMT problem while no exit constraint is considered on the job in the UPS problem. Though time allocation [18] has been studied in cooperative networks, time allocation among all the sources sharing a relay and/or destination node also imposes new challenges that have not been addressed by any existing solution.

\section{ALgorithm FOR M21-SRA-MMT}

This section mainly presents two relay assignment algorithms to achieve the max-min throughput. We also analyze the approximation performance of the proposed algorithm.

\subsection{Problem Formulation}

This section first formulates the M21-SRA-MMT problem as a mixed integer program. We define a binary variable $x_{i j}$, which is equal to 1 if relay node $r_{j} \in R$ is assigned to transmission pair $\Gamma_{i}$; and 0 otherwise. That is,

$$
x_{i j}=1 \text { or } 0, \forall s_{i} \in S, \forall r_{j} \in R
$$

Since each transmission pair (or source node) will use one relay node at most for cooperative communication, we have,

$$
\sum_{r_{j} \in R} x_{i j} \leq 1, \forall s_{i} \in S
$$

We also use $I_{i k} \in\{0,1\}$ to denote the $s$ - $d$ association. That is, if the source $s_{i}$ is associated with the destination $d_{k}, I_{i k}$ is 1 . Otherwise, $I_{i k}$ is 0 . Assume that each source $s_{i}$ will be allocated a fractional transmission time $t_{i} \leq 1$. For each destination node $d_{k}$, it may receive from (or be accessed by) several source nodes, and its total access time should not exceed one unit. That is,

$$
\sum_{s_{i} \in S} I_{i k} t_{i} \leq 1, \forall d_{k} \in D
$$

After relay assignment, the total transmission time of each relay node should not exceed one unit time either. So,

$$
\sum_{s_{i} \in S} t_{i} x_{i j} \leq 1, \forall r_{j} \in R
$$

Next, we use $C_{i j}$ to denote the capacity when source node $s_{i}$ communicates with destination node $d_{i^{\prime}}$ using relay node $r_{j}$. That is, $C_{i j}=C_{X F}\left(s_{i}, r_{j}, d_{i^{\prime}}\right)$ according to equations (1) or (2), where $X F$ denotes $A F$ or $D F$. Note that $C_{i, 0}$ denotes the capacity of transmission pair $\Gamma_{i}$ under the direct transmission scheme. That is, $C_{i, 0}=C_{D}\left(s_{i}, d_{i^{\prime}}\right)$ by equation (3). Now, we give the throughput expression of source node $s_{i}$ followed with two cases. On the one hand, source node $s_{i}$ selects one relay node $r_{j}$ for the cooperative communication. That is, $x_{i j}=1$. On the other hand, source node $s_{i}$ uses the direct transmission scheme. So, $\sum_{r_{j} \in R} x_{i j}=0$. As a result, the throughout achieved by each transmission pair $\Gamma_{i}$ is:

$$
B_{i}=\sum_{r_{j} \in R} C_{i j} t_{i} x_{i j}+C_{i, 0} t_{i}\left(1-\sum_{r_{j} \in R} x_{i j}\right)
$$

For the M21-SRA-MMT problem, we aim to maximize the minimum throughput of all source nodes. That is,

$$
\operatorname{Max}\left\{\min B_{i}, s_{i} \in S\right\}
$$

In this way, the optimization formulation of the M21-SRAMMT problem is in the form of a mixed integer program. The optimal solution to this problem is hard to find according to the following lemma.

Lemma 1: The M21-SRA-MMT problem is NP-hard. The proof of lemma 1 has been relegated to the appendix A.

\subsection{Algorithm Description}

Due to NP-hardness of the M21-SRA-MMT problem, we describe a rounding-based shared relay assignment algorithm, called RA-MM, to improve the minimum throughput of all the transmission pairs in the network. This algorithm consists of three steps, initialization, relay assignment and time allocation, respectively.

In the first step, the algorithm will construct a linear program $L P_{1}\left(t_{i j}\right)$ as a relaxation of M21-SRA-MMT. More specifically, the M21-SRA-MMT problem assumes that each source node only selects one relay node at most for cooperative communication. By relaxing this assumption, we can formulate the following linear program $L P_{1}\left(t_{i j}\right)$, which is solvable in polynomial time.

$$
\begin{array}{ll}
\text { Max } & B_{m} \\
\text { S.t. } & B_{i}=\sum_{r_{j} \in R^{+}} C_{i j} t_{i j} \geq B_{m}, \forall s_{i} \in S \\
& \sum_{s_{i} \in S} t_{i j} \leq 1, \forall r_{j} \in R \\
& \sum_{s_{i} \in S} \sum_{r_{j} \in R^{+}} I_{i k} t_{i j} \leq 1, \forall d_{k} \in D \\
& t_{i j} \in[0,1], \forall s_{i} \in S, \forall r_{j} \in R^{+}
\end{array}
$$

Note that, $t_{i j}$ is the transmission time of source node $s_{i}$ using relay node $r_{j}$ for cooperative communication. The second constraint says that the total transmission time fraction of each relay node $r_{j}$ can not be more than one unit. The third constraint means that the total access time fraction of each destination node $d_{k}$ should not exceed one unit. We obtain the optimal solution of $L P_{1}\left(t_{i j}\right)$, denoted by $\left\{\widetilde{t_{i j}}\right\}$, in which $\widetilde{t_{i j}}$ denotes the transmission time of source node $s_{i}$ using the relay node $r_{j}$ for CC or DT (note that in this 
relaxed version, we allow a source to use more than one relay node). Under this solution, for each source node $s_{i}$, its total transmission time is denoted by $T_{i}=\sum_{r_{j} \in R^{+}} \widetilde{t_{i j}} \leq 1$ according to the time constraint on each destination node, and the throughput is $B_{i}=\sum_{r_{j} \in R^{+}} C_{i j} \widetilde{t_{i j}}$. Moreover, the minimum throughput of all source nodes is denoted by $B_{o}$. Since $L P_{1}\left(t_{i j}\right)$ is the formulation of the relaxed M21-SRAMMT problem, in which each source node may use several relay nodes for cooperative transmission, $B_{o}$ is an upper-bound on the minimum throughput performance of M21-SRA-MMT.

In the second step, the algorithm will assign at most one relay for each source node using the rounding mechanism. We select a parameter $\alpha=\sqrt{2}+1$, which will lead to the smallest approximation ratio, and be explained later. This step first defines a variable $t_{i j}^{\prime}$ for each source node $s_{i}$ and relay node $r_{j}$. Given the variables $B_{i}$ and $T_{i}$ for each source $s_{i}$, if $B_{i}>\alpha C_{i j} T_{i}, t_{i j}^{\prime}=0$. Otherwise, $t_{i j}^{\prime}=\widetilde{t_{i j}}$. Based on the solution $\left\{t_{i j}^{\prime}\right\}$, the throughput of each source $s_{i}$ becomes $B_{i}^{\prime}=\sum_{r_{j} \in R^{+}} C_{i j} t_{i j}^{\prime}$. Next, we define another variable $\widetilde{x_{i j}}=\frac{C_{i j} t_{i j}^{\prime}}{B_{i}^{\prime}}$, which denotes the percentage of throughput $B_{i}^{\prime}$ contributed by a relay $r_{j}$ for a given source $s_{i}$. Accordingly, $\sum_{r_{j} \in R^{+}} \widetilde{x_{i j}}=\sum_{r_{j} \in R^{+}} \frac{C_{i j} t_{i j}^{\prime}}{B_{i}^{\prime}}=1$. For each relay $r_{j}$, $\sum_{s_{i} \in S} t_{i j}^{\prime} \leq \sum_{s_{i} \in S} \widetilde{t_{i j}} \leq 1$. It follows that $\sum_{s_{i} \in S} \frac{B_{i}^{\prime} \widetilde{x_{i j}}}{C_{i j}} \leq 1$ after substituting $t_{i j}^{\prime}$ by $\frac{B_{i}^{\prime} \widetilde{x_{i j}}}{C_{i j}}$. Thus, we know that $\left\{\widetilde{x_{i j}}\right\}$ is a solution for the linear program $L P_{2}\left(x_{i j}\right)$ as follows:

$$
\begin{aligned}
\text { S.t. } & \sum_{r_{j} \in R^{+}} x_{i j}=1, \forall s_{i} \in S \\
& \sum_{s_{i} \in S} \frac{B_{i}^{\prime}}{C_{i j}} x_{i j} \leq 1, \forall r_{j} \in R \\
& x_{i j} \in[0,1], \forall s_{i} \in S, \forall r_{j} \in R^{+}
\end{aligned}
$$

Then, an integer solution $\left\{\widehat{x_{i j}}\right\}$ can be obtained from $\left\{\widetilde{x_{i j}}\right\}$ by the rounding procedure [19]. Under the solution $\left\{\widehat{x_{i j}}\right\}$, if $\widehat{x_{i j}}$ is 1 , this means that the relay node $r_{j}$ is assigned to source node $s_{i}$ for cooperative communication. Otherwise, source $s_{i}$ will use the direct transmission.

In the third step, we will determine the transmission time for each source node $s_{i}$. Under the solution $\left\{\widehat{x_{i j}}\right\}$, the algorithm will check all the relay nodes as follows. For each relay $r_{j} \in R$, the source nodes that share this relay for cooperative transmission are considered to be in set $S_{r_{j}}$. We compute its total time as $\delta_{j}=\sum_{s_{i} \in S_{r_{j}}} T_{i}$. If $\delta_{j} \leq 1, t_{i}=T_{i}$. Otherwise, let $t_{i}^{\prime}=\frac{B_{i}^{\prime}}{(1+\alpha) C_{i j}}$ and $\delta_{j}^{0}=\sum_{s_{i} \in S_{r_{j}}} t_{i}^{\prime}$. We set the transmission time of each source node $s_{i} \in S_{r_{j}}$ as $t_{i}=\min \left\{T_{i}, \frac{t_{i}^{\prime}}{\delta_{j}^{0}}\right\}$. A more detailed description of RA-MM is given in Algorithm 1, where $R\left(s_{i}\right)$ denotes the assigned relay for the source node $s_{i}$.

\subsection{Approximation Performance Analysis}

Before analyzing the worst-case approximation performance of the RA-MM algorithm, we first show that:

Lemma 2: for each source node $s_{i}, B_{i}^{\prime} \geq\left(1-\frac{1}{\alpha}\right) B_{o}$.

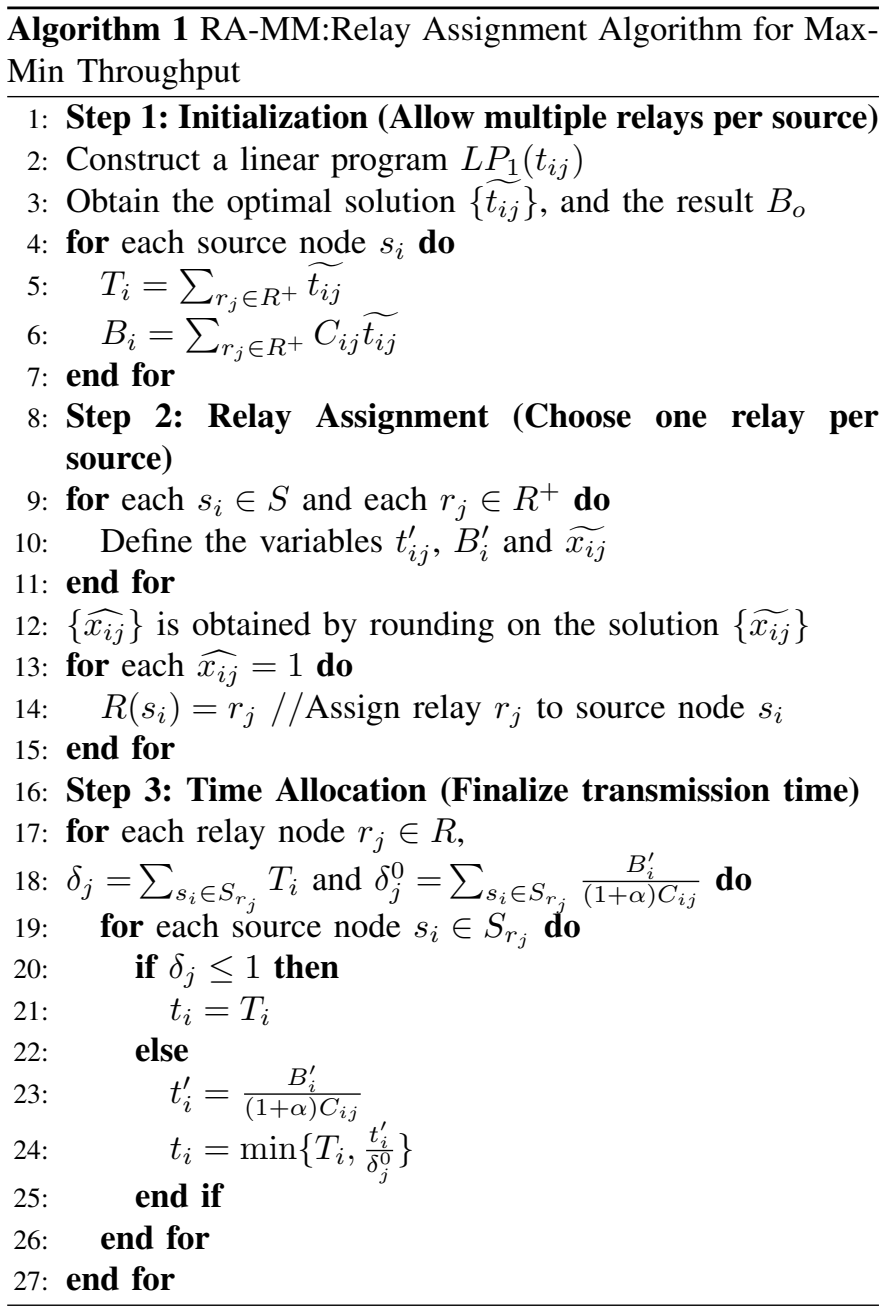

Proof: By the definition of variable $B_{i}^{\prime}$ in the second step of the RA-MM algorithm, for each source $s_{i}$, it follows that:

$$
\begin{aligned}
B_{i} & =\sum_{r_{j} \in R^{+}} C_{i j} \widetilde{t_{i j}}=\sum_{t_{i j}^{\prime}>0} C_{i j} \widetilde{t_{i j}}+\sum_{t_{i j}^{\prime}=0} C_{i j} \widetilde{t_{i j}} \\
& =B_{i}^{\prime}+\sum_{t_{i j}^{\prime}=0} C_{i j} \widetilde{t_{i j}} \leq B_{i}^{\prime}+\frac{B_{i}}{\alpha T_{i}} \sum_{r_{j} \in R^{+}} \widetilde{t_{i j}} \\
& =B_{i}^{\prime}+\frac{B_{i}}{\alpha T_{i}} T_{i} \leq B_{i}^{\prime}+\frac{B_{i}}{\alpha}
\end{aligned}
$$

This indicates that $B_{i}^{\prime} \geq\left(1-\frac{1}{\alpha}\right) B_{i} \geq\left(1-\frac{1}{\alpha}\right) B_{o}$.

Now, we analyze the approximation performance for the RA-MM algorithm.

Theorem 3: the RA-MM algorithm can reach the approximate factor of $3+2 \sqrt{2}$ for the M21-SRA-MMT problem.

Proof: By the definition of variables $B_{i}^{\prime}$ and $\widetilde{x_{i j}},\left\{\widetilde{x_{i j}}\right\}$ is a solution of the linear program $L P_{2}\left(x_{i j}\right)$ as in equation (13). In the second constraint of $L P_{2}\left(x_{i j}\right)$,

$$
\frac{B_{i}^{\prime}}{C_{i j}} \leq \frac{B_{i}}{C_{i j}} \leq \alpha T_{i} \leq \alpha
$$

Then, a fractional solution $\left\{\widetilde{x_{i j}}\right\}$ can be rounded to a $0-1$ solution $\left\{\widehat{x_{i j}}\right\}$ for the integer linear program $I P_{3}\left(x_{i j}\right)$ as:

$$
\begin{array}{ll}
\text { S.t. } & \sum_{r_{j} \in R^{+}} x_{i j}=1, \forall s_{i} \in S \\
& \sum_{s_{i} \in S} \frac{B_{i}^{\prime}}{C_{i j}} x_{i j} \leq 1+\alpha, \forall r_{j} \in R \\
& x_{i j} \in\{0,1\}, \forall s_{i} \in S, \forall r_{j} \in R^{+}
\end{array}
$$


where $\widehat{x_{i j}}$ is 1 , if source node $s_{i}$ will select relay node $r_{j}$ for cooperative transmission. Otherwise, $\widehat{x_{i j}}$ is 0 . In the third step, each source node $s_{i}$ will be allocated a transmission time $t_{i}$. Let $t_{i}^{\prime}=\frac{B_{i}^{\prime}}{(1+\alpha) C_{i j}}$. We consider the following two cases:

1) $\delta_{j} \leq 1 . t_{i}=T_{i}$, and $t_{i}^{\prime}=\frac{B_{i}^{\prime}}{(1+\alpha) C_{i j}} \leq \frac{\alpha T_{i}}{1+\alpha}<T_{i}$. So, $t_{i}^{\prime}<t_{i}$

2) $\delta_{j}>1$. Then, $t_{i}=\min \left\{T_{i}, \frac{t_{i}^{\prime}}{\delta_{j}^{0}}\right\}$. Since $\delta_{j}^{0}=\sum_{s_{i} \in S_{r_{j}}} t_{i}^{\prime}$ $=\sum_{s_{i} \in S_{r_{j}}} \frac{B_{i}^{\prime}}{(1+\alpha) C_{i j}} \leq \frac{1+\alpha}{1+\alpha}=1, t_{i}^{\prime} \leq \min \left\{T_{i}, \frac{t_{i}^{\prime}}{\delta_{j}^{0}}\right\}=t_{i}$.

Combining the above two cases, we know that $t_{i}^{\prime} \leq t_{i}$. The final throughput of each source $s_{i}$, denoted by $\overline{B_{i}}$, is:

$$
\begin{aligned}
\overline{B_{i}} & =\sum_{r_{j} \in R^{+}} C_{i j} \widehat{x_{i j}} t_{i} \geq \sum_{r_{j} \in R^{+}} C_{i j} \widehat{x_{i j}} t_{i}^{\prime} \\
& =\sum_{r_{j} \in R^{+}} C_{i j} \widehat{x_{i j}} \frac{B_{i}^{\prime}}{(1+\alpha) C_{i j}} \\
& =\frac{B_{i}^{\prime}}{1+\alpha} \\
& \geq \frac{1}{1+\alpha}\left(1-\frac{1}{\alpha}\right) B_{o}
\end{aligned}
$$

We find the best parameter $\alpha$ to achieve a throughput as close to the optimum as possible, that is, to make $\frac{1}{1+\alpha}\left(1-\frac{1}{\alpha}\right)$ as large as possible. This results in $\alpha=\sqrt{2}+1$. So

$$
\overline{B_{i}} \geq \frac{1}{1+\sqrt{2}+1}\left(1-\frac{1}{\sqrt{2}+1}\right) B_{o}=\frac{1}{3+2 \sqrt{2}} B_{o}
$$

As a result, we can conclude that the RA-MM algorithm can reach the approximation performance of $3+2 \sqrt{2}$ for the M21-SRA-MMT problem. The theorem is proved.

\subsection{An Improved RA-MM Algorithm (IRA-MM)}

In the RA-MM algorithm, the third (i.e., time allocation) step determines the transmission time for all the source nodes in a proportional manner. Though this method can provide the approximate performance guarantee (in the worst case), it does not care for the different capacities among these transmission pairs. Thus, this section designs an improved algorithm, called IRA-MM, so as to enhance its minimum throughput of all the sources compared with that by the RA-MM algorithm.

After the second step of the RA-MM algorithm, each source node $s_{i}$ has determined the relay assignment. For simplicity, a set of source nodes that share the relay $r_{j} \in R$ is denoted by $S_{r_{j}}=\left\{s_{1}, \ldots, s_{k}\right\}$, with $\left|S_{r_{j}}\right|=k$. The basic idea of IRAMM is to let selected sources use the relay without committing the relay to all the $k$ sources so as to reach the almost same throughput among these sources. More specifically, we compute its total transmission time as $\delta_{j}=\sum_{s_{i} \in S_{r_{j}}} T_{i}$. If $\delta_{j} \leq 1$, we also set $t_{i}=T_{i}$ for each source $s_{i} \in S_{r_{j}}$. In this case, the minimum throughput of all sources in $S_{r_{j}}$ will not be improved. Otherwise (i.e., if $\delta_{j}>1$ ), the algorithm ranks all source nodes in a decreasing order of the throughput under the direct transmission (denoted by $C_{i, 0} T_{i}$ for each source $s_{i}$ ). Without loss of generality, assume that $C_{1,0} T_{1} \geq C_{2,0} T_{2} \geq$ $\ldots \geq C_{k, 0} T_{k}$. We compute the minimum throughput $m t_{q}$ when the first $q \geq 0$ nodes use direct transmission, and the rest of the $k-q$ sources, denoted by $S_{r_{j}}^{q}=\left\{s_{q+1}, \ldots, s_{k}\right\}$, will use the relay to boost their throughputs (note that the sum of the transmission time from these $k-q$ sources should be less than 1). Among the first $q$ sources, due to the fact that the sources are already sorted in the order of a decreasing throughput, the minimum throughput is $C_{q, 0} T_{q}$ from source $s_{q}$. Among the rest of $k-q$ sources, let $t_{i}^{\prime}=\frac{B_{i}^{\prime}}{(1+\alpha) C_{i j}}$, and $\delta_{j}^{q}=\sum_{s_{i} \in S_{r_{j}}^{q}} t_{i}^{\prime}(<1)$. We set $t^{\prime \prime}{ }_{i}=\min \left\{T_{i}, \frac{t_{i}^{\prime}}{\delta_{j}^{q}}\right\}$ for each source $s_{i}$. The minimum throughput of the sources in $S_{r_{j}}^{q}$ is $b_{q}=\min \left\{C_{i j} t^{\prime \prime}{ }_{i}, s_{i} \in S_{r_{j}}^{q}\right\}$. So, $m t_{q}$ can be expressed by:

$$
m t_{q}=\min \left\{C_{q, 0} T_{q}, b_{q}\right\}, 1 \leq q<k
$$

Finally, the algorithm determines the optimal $q$ value, denoted by $q_{0}$, so that $m t_{q_{0}}=\max \left\{m t_{q}, 1 \leq q<k\right\}$. The outcome of the algorithm is thus as follows: the first $q_{0}$ sources use the DT, and the rest $k-q_{0}$ source nodes use the cooperative communication with the help of relay node $r_{j}$. The IRA-MM algorithm is formally described in Algorithm 2, where $R\left(s_{i}\right)$ denotes the assigned relay for the source node $s_{i}$.

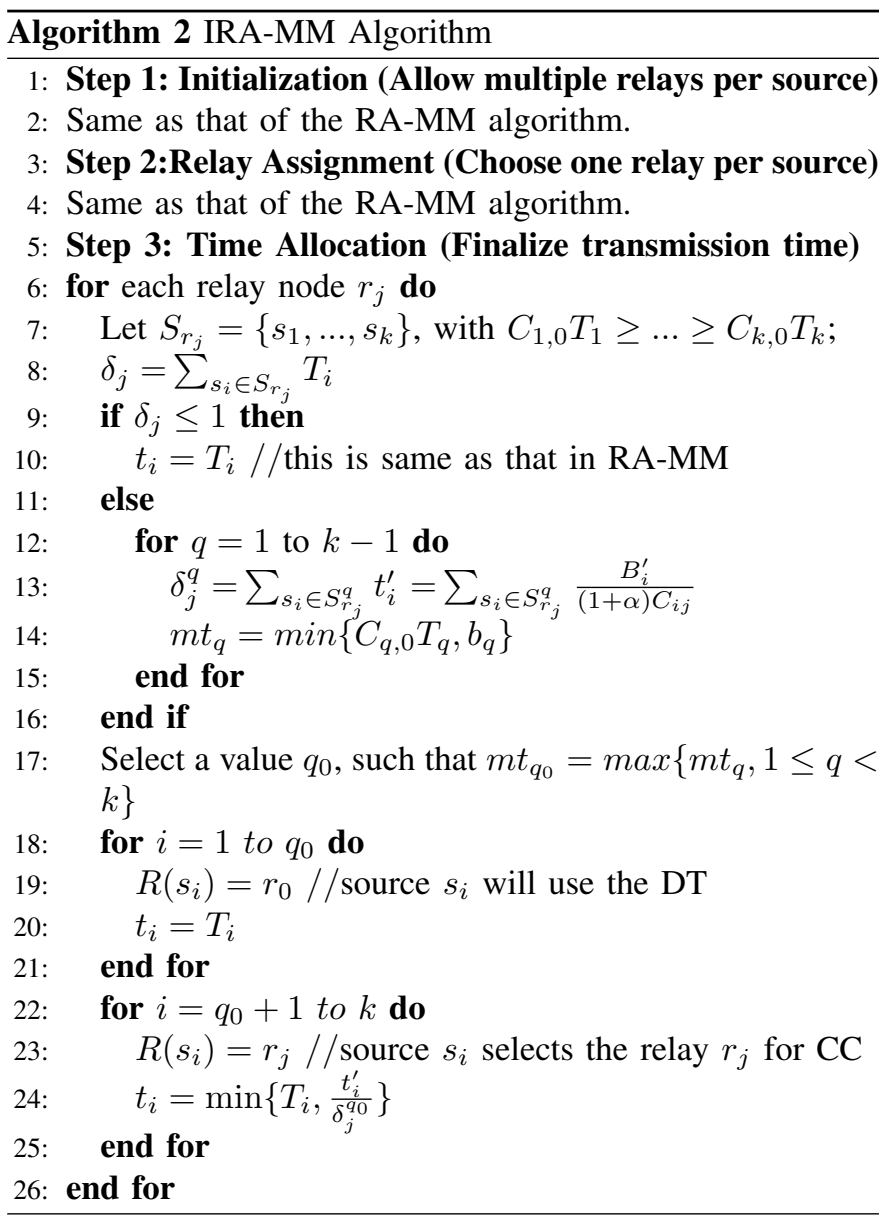

According to the above description, the minimum throughput should be no less than that obtained by RA-MM after the second step. Thus, the IRA-MM algorithm improves the MMT performance upon RA-MM. Therefore,

Theorem 4: The IRA-MM algorithm can reach the approximate factor of $3+2 \sqrt{2}$ for the M21-SRA-MMT problem.

\section{ALGORITHM FOR M21-SRA-MTT}

The previous section tries to maximize the minimum throughput of all the source nodes. However, in some situations, 
the total network throughput is much critical for wireless systems. Thus, this section mainly studies the shared relay assignment problem for M21 traffic that can maximize the total throughput, termed M21-SRA-MTT.

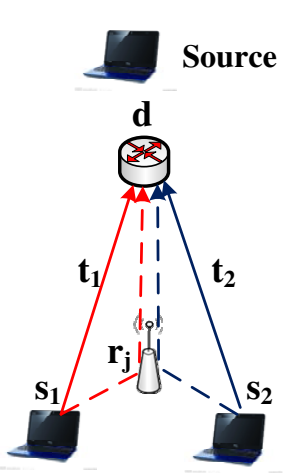

(a)

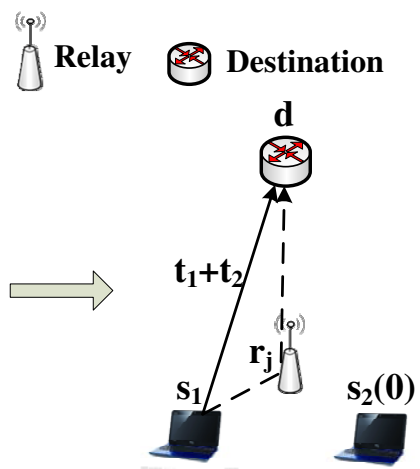

(b)
Fig. 2: Illustration of Anycast with Software Defined Networks

\subsection{Motivation and Problem Definition}

Let us now consider the following case, where two sources $s_{1}$ and $s_{2}$ communicate with the same destination node, and are allocated with transmission time $t_{1}$ and $t_{2}$, respectively. Assume that they share the same relay node $r_{j}$, as shown in Fig.2(a). The total throughput of two sources is $C_{1, j} t_{1}+C_{2, j} t_{2}$. Without loss of generality, assume that $C_{1, j}>C_{2, j}$. If we change the transmission time allocation to be $t_{1}^{\prime}=t_{1}+t_{2}$ and $t_{2}^{\prime}=0$, respectively, then, the total throughput will be $C_{1, j} t_{1}^{\prime}=C_{1, j}\left(t_{1}+t_{2}\right)>C_{1, j} t_{1}+C_{2, j} t_{2}$, illustrated in Fig.2. Though the total throughput will be improved, source node $s_{2}$ is not able to obtain any opportunity for transmission at all. This example shows that in general, some source nodes may become starved in the network when one blindly pursues the maximum total throughout, which is not only undesirable but also may degenerate the problem into dedicated relay assignment and 121 traffic. Thus, this section mainly studies the max-total throughput problem under a certain fairness constraint among all the source nodes. In particular, we require that for a given destination $d$, all its sources will have an equal access time, so that the starvation will be avoided. That is, the common destination will devote an equal amount of access time to each of its $k$ associated sources. In other words, each source $s_{i}$ will be allocated a transmission time $t_{i}=\frac{1}{k}$ to communicate with the common destination $d$ (using the DT or CC schemes). We also take the same assumption that each source node will select one relay node at most for cooperative communication as in the definition of M21-SRAMMT. After relay assignment, each source node $s_{i}$ will obtain the achievable throughput $B_{i}$. The objective of the M21-SRAMTT problem is to maximize the total throughput of all the sources in the network. That is, $\operatorname{Max} \sum_{s_{i} \in S} B_{i}$.

Now, we give the formulation for the M21-SRA-MTT problem below. In the formulation, the Boolean variable $x_{i j}$ denotes whether the source node $s_{i}$ dominates the relay node $r_{j}$ or not, and $t_{i}$ is the pre-allocated transmission time for source node $s_{i}$. In addition, in the M21-SRA-MTT formalization, $t_{i}$ is a constant, and $t_{i}=\frac{1}{k}$ if source node $s_{i}$ shares the same destination with $k-1$ other source nodes.

$$
\begin{array}{ll}
\text { Max } & B_{m}=\sum_{s_{i} \in S} \sum_{r_{j} \in R^{+}} C_{i j} t_{i} x_{i j} \\
\text { S.t. } & \sum_{r_{j} \in R^{+}} x_{i j}=1, \forall s_{i} \in S \\
& \sum_{s_{i} \in S} t_{i} x_{i j} \leq 1, \forall r_{j} \in R \\
& x_{i j} \in\{0,1\}, \forall s_{i} \in S, \forall r_{j} \in R^{+} .
\end{array}
$$

The first constraint says that the total time fraction of each source node is $100 \%$. The second constraint says that the total time fraction of each relay node $r_{j}$ is not more than 1 . In this way, the M21-SRA-MTT problem can be formulated into a mixed integer program. Although we do not prove the NPHarness of M21-SRA-MTT in this paper, it is suffice to say that it is difficult to obtain the optimal solution to this problem. Accordingly, we will proceed with an approximate algorithm next.

\subsection{Algorithm Description}

Here we describe a relay assignment algorithm, called RAMT, to maximize the total throughput of all the source nodes with time-wise fairness. This algorithm consists of two main steps, relay pre-assignment and relay adjustment, respectively.

The first step of relay pre-assignment will start by allowing multiple relays per source node and then narrow down to one relay at most per source using the rounding method. To do so, the algorithm constructs a linear program $L P_{4}\left(x_{i j}\right)$, which is similar to $L P_{1}\left(t_{i j}\right)$, as follows:

$$
\begin{array}{ll}
\operatorname{Max} & B_{m}=\sum_{s_{i} \in S} \sum_{r_{j} \in R^{+}} C_{i j} t_{i} x_{i j} \\
\text { S.t. } & \sum_{r_{j} \in R^{+}} x_{i j}=1, \forall s_{i} \in S \\
& \sum_{s_{i} \in S} t_{i} x_{i j} \leq 1, \forall r_{j} \in R \\
& x_{i j} \in[0,1], \forall s_{i} \in S, \forall r_{j} \in R^{+}
\end{array}
$$

where $x_{i j}$ denotes the fraction of time in which the source node $s_{i}$ dominates the relay node $r_{j}$, and $t_{i}$ is the pre-allocated transmission time for source node $s_{i}$. The first constraint says that the total time fraction of each source node is $100 \%$. The second constraint says that the total time fraction of each relay node $r_{j}$ is not more than 1 .

We can solve $L P_{4}\left(x_{i j}\right)$ in polynomial time, and obtain its solution $\left\{\widetilde{x_{i j}}\right\}$. The optimal result of $L P_{4}\left(x_{i j}\right)$ can be expressed by $B_{t}=\sum_{s_{i} \in S} \sum_{r_{j} \in R^{+}} C_{i j} t_{i} \widetilde{x_{i j}}$. Since $L P_{4}\left(x_{i j}\right)$ is the formalization of the relaxed M21-SRA-MTT problem, in which each source node may use several relay nodes for cooperative transmission, $B_{t}$ is an upper-bound on the total throughput performance of M21-SRA-MTT. Then, an integer solution $\left\{\overline{x_{i j}}\right\}$ is obtained from $\left\{\widetilde{x_{i j}}\right\}$ by rounding [20]. Under the solution $\left\{\overline{x_{i j}}\right\}$, if $\overline{x_{i j}}$ is 1 , the source $s_{i}$ will select the relay $r_{j}$, and otherwise, it will use the direct transmission scheme. Let the total throughput achieved in this way be denoted by $B_{t}^{\prime}$, which can be expressed by $B_{t}^{\prime}=\sum_{s_{i} \in S} \sum_{r_{j} \in R^{+}} C_{i j} t_{i} \overline{x_{i j}}$. According to [20], this rounding procedure will not result in a lower total throughput than $B_{t}$. Therefore, $B_{t}^{\prime}$ is also an upper-bound for the M21-SRA-MTT problem. For simplicity, we use $S_{r_{j}}$ to denote a set of sources that share relay $r_{j}$. 
Note that, $B_{t}^{\prime}$ consists of two parts: the sum of the throughout from the source nodes who use the direct transmission scheme, denoted by $B\left(S_{r_{0}}\right)=\sum_{s_{i} \in S_{r_{0}}} C_{i, 0} t_{i}$, and the sum of the throughout from the source nodes who use the cooperative communication with the help of a relay $r_{j}\left(\neq r_{0}\right)$, denoted by $\sum_{r_{j} \in R} B\left(S_{r_{j}}\right)=\sum_{s_{i} \in S_{r_{j}}} C_{i j} t_{i}$. That is,

$$
B_{t}^{\prime}=\sum_{s_{i} \in S_{r_{0}}} C_{i, 0} t_{i}+\sum_{r_{j} \in R} \sum_{s_{i} \in S_{r_{j}}} C_{i j} t_{i}
$$

Note that although solution $\left\{\overline{x_{i j}}\right\}$ will specify (at most) one relay per source node, multiple source nodes may share one relay. In particular, the total transmission time of each relay node $r_{j} \in R$ is $\delta_{j}=\sum_{s_{i} \in S} t_{i} \overline{x_{i j}} \leq 2$ (by the rounding method [20]), but the time constraint on some relay nodes may not be satisfied (as $\delta_{j}$ may be larger than 1). Accordingly, the second step should adjust the relay assignment as follows so as to satisfy the time constraint on each relay node.

For ease of expression, the set of sources that share the relay $r_{j}$ is denoted by $S_{r_{j}}=\left\{s_{1}, \ldots, s_{k}\right\}$, with $k=\left|S_{r_{j}}\right|$. We will order the sources in $S_{r_{j}}$ in a descending order of $t_{i}$, and use $C_{i j}$ as a tiebreaker. In other words, each source $s_{i}$ will be assigned a weight, which is based on $t_{i}$ with $C_{i j}$ used for tiebreaker. For simplicity, we loosely define $w\left(s_{i}\right)=\left(t_{i}, C_{i j}\right)$, and assume that $w\left(s_{1}\right) \geq \ldots \geq w\left(s_{k}\right)$. The basic idea is to first partition this set $S_{r_{j}}$ into as few subsets as possible such that the sum of transmission time by the sources in each subset does not exceed one. As to be shown later, the number of such subsets would be at least 2 but at most 3 . The partitioned subsets can be denoted by $V_{1}, V_{2}$ and $V_{3}$ (if applicable). For each source set $V_{q}$ with $1 \leq q \leq 3$, we compute its total throughput as $B\left(V_{q}\right)=\sum_{s_{i} \in V_{q}} C_{i j} t_{i}$. The algorithm then selects the one with the maximum total throughput, denoted by $V_{q^{\prime}}$, where $q^{\prime}$ is either 1,2 or 3 , and assign the relay $r_{j}$ to the source nodes in the set $V_{q^{\prime}}$. Other source nodes in the set $S_{r_{j}}-V_{q^{\prime}}$ will use the direct transmission scheme. A formal description of the RA-MT algorithm is shown in Algorithm 3.

Note that, for each relay node $r_{j}$ (where $r_{j} \neq r_{0}$ ), assume without loss of generality that the second step of the algorithm picks $q^{\prime}=1$ (because, $B\left(V_{1}\right) \geq B\left(V_{2}\right)$ and $B\left(V_{1}\right) \geq B\left(V_{3}\right)$ ). Then, after the second step of the RA-MT algorithm, the total throughput of all the sources in the set $S_{r_{j}}$ is expressed by $B^{*}\left(S_{r_{j}}\right)=B\left(V_{1}\right)+\sum_{s_{i} \in V_{2}} C_{i, 0} t_{i}+\sum_{s_{i} \in V_{3}} C_{i, 0} t_{i}$. Since the source nodes in $V_{2}$ and $V_{3}$ can no longer use the relay $r_{j}$, $B^{*}\left(S_{r_{j}}\right)$ may be less than $B\left(S_{r_{j}}\right)$. Nevertheless, the reduction will be no greater than $\frac{2}{3}$. That is,

$$
B^{*}\left(S_{r_{j}}\right) \geq B\left(V_{1}\right) \geq \frac{1}{3} \sum_{s_{i} \in S_{r_{j}}} C_{i j} t_{i}=\frac{1}{3} B\left(S_{r_{j}}\right)
$$

Finally, if we consider all the relays in set $R$, we have:

$$
\begin{aligned}
\sum_{r_{j} \in R} B^{*}\left(S_{r_{j}}\right) & \geq \frac{1}{3} \sum_{r_{j} \in R} \sum_{s_{i} \in S_{r_{j}}} C_{i j} t_{i} \\
& =\frac{1}{3} \sum_{r_{j} \in R} B\left(S_{r_{j}}\right)
\end{aligned}
$$

\subsection{Approximation Performance Analysis}

As mentioned earlier, at the end of the first step, each source node will be pre-assigned a relay node. The set of source nodes that select the relay node $r_{j}$ is denoted by $S_{r_{j}}$. By the rounding method [20], we know the transmission time of each

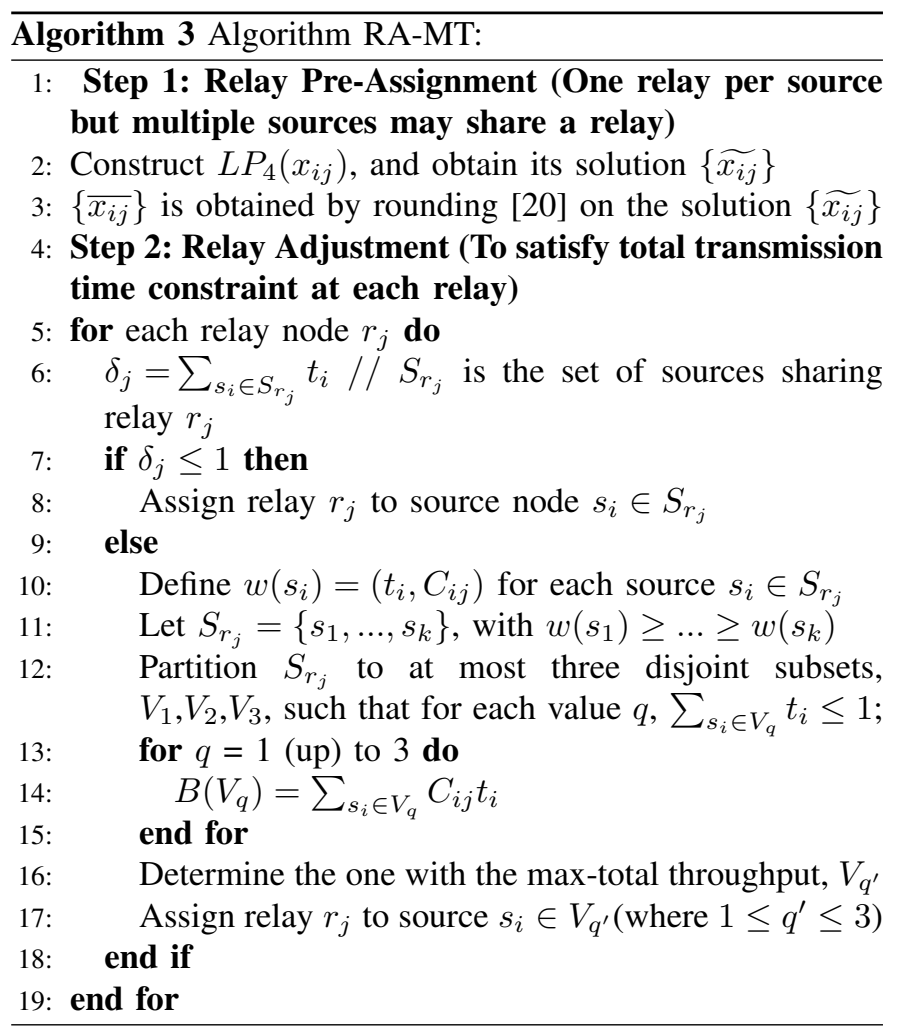

relay $r_{j}$ is $\delta_{j}=\sum_{s_{i} \in S_{r_{j}}} t_{i} \overline{x_{i j}} \leq 2$. If $\delta_{j} \leq 1$, there is no need for relay adjustment. We just consider the case of $\delta_{j}>1$, and prove that:

Lemma 5: The second step of the RA-MT algorithm will partition the node set $S_{r_{j}}$ into at most three subsets.

Proof: Assume by contradiction that three source subsets, denoted by $V_{1}, V_{2}$ and $V_{3}$, cannot cover the set $S_{r_{j}}$. The sum of transmission time by the sources in each subset $V_{i}$ is described as $\beta_{i}=\sum_{s_{l} \in V_{i}} t_{l}$, where $i=1,2,3$. We consider two cases:

1) $\beta_{1}=1$. Then, we set $V_{2}=S_{r_{j}}-V_{1}$, with $\beta_{2}=\delta_{j}-\beta_{1} \leq$ 1. So, two subsets $V_{1}$ and $V_{2}$ can cover the node set $S_{r_{j}}$. 2) $\beta_{1}<1$. Let $q_{i}=\left|V_{i}\right|, i=1,2,3$. For ease of expression, $t_{1^{\prime}}=t_{q_{1}+1}, t_{2^{\prime}}=t_{q_{1}+q_{2}+1}$ and $t_{3^{\prime}}=t_{q_{1}+q_{2}+q_{3}+1}$. According to the algorithm, although the total transmission time by each subset does not exceed one, it would not after including the subsequent source node in the sorted list. So, $\beta_{i}+t_{i^{\prime}}>1$, for $i=1,2,3$

Thus,

$$
\beta_{1}+t_{1^{\prime}}+\beta_{2}+t_{2^{\prime}}+\beta_{3}+t_{3^{\prime}}>3
$$

Since each of the sources sharing a common destination will be allocated an equal amount of access time in the form of $\frac{1}{k}$ for some $k$ values, the possible transmission time of each source $s_{i}$ is $t_{i} \in\left\{1, \frac{1}{2}, \frac{1}{3}, \ldots\right\}$. Let us consider the value of $t_{1^{\prime}}$.

a) $t_{1^{\prime}}=1$. By assumption, $t_{1^{\prime}}=1 \leq t_{1}$. We have $t_{1}=1$ too (i.e., $q_{1}$ must be 1 , and there are only two source nodes $s_{1}$ and $s_{2}$ in $S_{r_{j}}$ ). This thus becomes the same as case 1 .

b) $t_{1^{\prime}}=\frac{1}{2}$. Given the sorted list of the sources, it follows that we have either $t_{1}=1$ or $t_{1}=t_{2}=\frac{1}{2}$. In either case, this also falls into case 1 . 
c) $t_{1^{\prime}} \leq \frac{1}{3}$, which also indicates that $t_{3^{\prime}} \leq t_{2^{\prime}} \leq t_{1^{\prime}} \leq \frac{1}{3}$. According to the assumption in the contradiction proof, and equation (24), we have:

$$
\begin{aligned}
\delta_{j} & =\sum_{s_{i} \in S_{r_{j}}} t_{i} \\
& >\sum_{s_{i} \in V_{1} \cup V_{2} \cup V_{3}} t_{i} \\
& =\beta_{1}+\beta_{2}+\beta_{3}>3-t_{1^{\prime}}-t_{2^{\prime}}-t_{3^{\prime}}>3-3 t_{1^{\prime}} \\
& \geq 3-3 \cdot \frac{1}{3}=2
\end{aligned}
$$

This contradicts with the previously mentioned fact that by rounding, $\delta_{j} \leq 2$. Hence, there can be at most 3 subsets.

Based on this lemma, we analyze the approximation performance of the RA-MT algorithm.

Theorem 6: the RA-MT algorithm can reach the approximation performance of 3 for the M21-SRA-MTT problem.

Proof: Recall that at the end of the first step of relay pre-assignment in RA-MT, the total throughput $B_{t}^{\prime}$ is given in equation (21), which is no less than $B_{t}$ according to [20].

Additionally, at the end of the RA-MT algorithm, the total throughput achieved, denoted by $B_{M T}$, is the sum of the first term in the left-hand side of equation (21), and $\sum_{r_{j} \in R} B^{*}\left(S_{r_{j}}\right)$ in equation (23). That is,

$$
\begin{aligned}
B_{M T} & =\sum_{s_{i} \in S_{r_{0}}} C_{i, 0} t_{i}+\sum_{r_{j} \in R} B^{*}\left(S_{r_{j}}\right) \\
& \geq \frac{1}{3} \sum_{s_{i} \in S_{r_{0}}} C_{i, 0} t_{i}+\frac{1}{3} \sum_{r_{j} \in R} \sum_{s_{i} \in S_{r_{j}}} C_{i j} t_{i} \\
& =\frac{1}{3} B_{t}^{\prime}
\end{aligned}
$$

In other words, the RA-MT algorithm can reach the approximate factor of 3 for the M21-SRA-MTT problem.

\section{Average-Case Performance Results}

This section mainly presents the numerical results from simulations to demonstrate the average-case throughput efficiency of the proposed algorithms. The simulations are run using the network simulator OMNet++ (Objective Modular Network Test-bed in $\mathrm{C}++)$. We assume the reliable transmission in the wireless networks using the built-in MAC of OMNet++ (which uses CSMA) by setting packet loss ratio to zero.

\subsection{Simulation Setting and Measurements}

In the simulations, we consider a wireless network whose nodes are randomly deployed in an area of $800 \mathrm{~m} \times 600 \mathrm{~m}$. That is, given a rectangle area, we randomly generate a position coordinate (according to a uniform distribution of both $x$ and $y$ coordinates), and place a node at this location. By default, the number of destination nodes and relays are 5 for each, while the number of sources (which should be more than that of destination nodes in M21 traffic) is set to 40. We will observe the impact of varying the numbers of source nodes, the numbers of relay nodes, and area sizes, etc., on the performances of minimum throughput and total throughput, respectively.

For most of the other parameters, we adopt the same settings as those in the ORA simulations [1]. For example, we assume that the bandwidth of each wireless channel is $W=22 M H Z$.

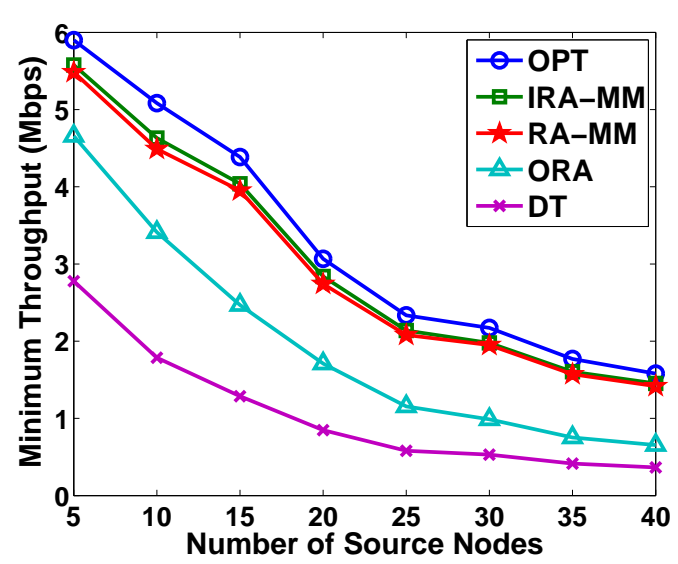

(a) $n_{r}=5$

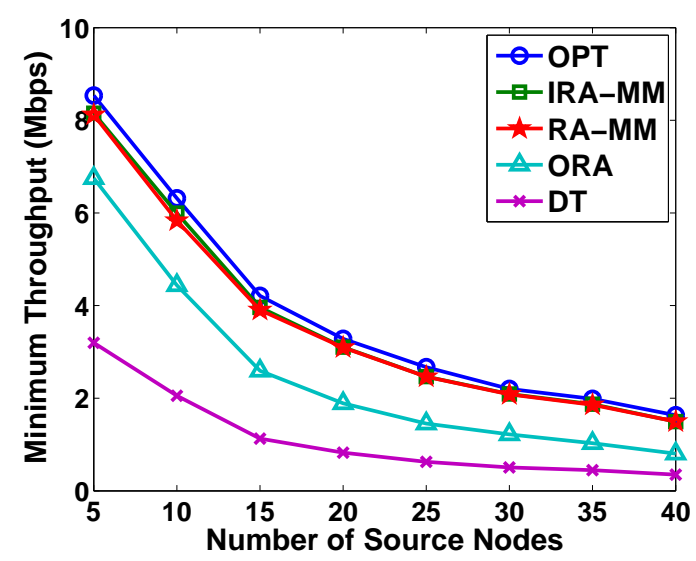

(b) $n_{r}=10$

Fig. 3: Minimum Throughput vs. Number of Source Nodes

The signal-noise-ratio from node $s$ to node $d$ is derived by $S N R_{s d}=\frac{P_{s} * h_{s d}^{2}}{\sigma_{d}^{2}}$, where $P_{s}$ is the transmission power of node $s, h_{s d}$ is the effect of path-loss, shadowing and fading between nodes $s$ and $d$, and $\sigma_{d}^{2}$ is the variance of the background noise at node $d$. For simplicity, we assume that $h_{s d}$ only includes the path loss component between nodes $s$ and $d$, and is expressed as $h_{s d}^{2}=|s d|^{-4}$, where $|s d|$ is the distance (in meters) between two nodes. The maximal transmission power is set to $1 W$ on both sources and relay nodes. For the $A W G N$ channel, it is assumed that the variance of noise is $10^{-10} \mathrm{~W}$ at all nodes [1]. Also, each relay node works in the $A F$ mode.

We evaluate our algorithms by comparing their max-min or max-total throughput performance with the following benchmark approaches. The first one is the direct transmission scheme. When applying direct transmission to M21 traffic, we allocate each source sharing the same destination an equal amount of time (whether the objective is max-min throughput or max-total throughput). The other is the ORA algorithm [1] [15], which was designed for max-min throughout, and hence will be compared with our RA-MM and IRA-MM only. As ORA does not at all consider time allocation among the source nodes for M21 traffic, we extend it by also allocating an equal amount of time to each of the source nodes sharing the same destination (while using a dedicated relay if any). 


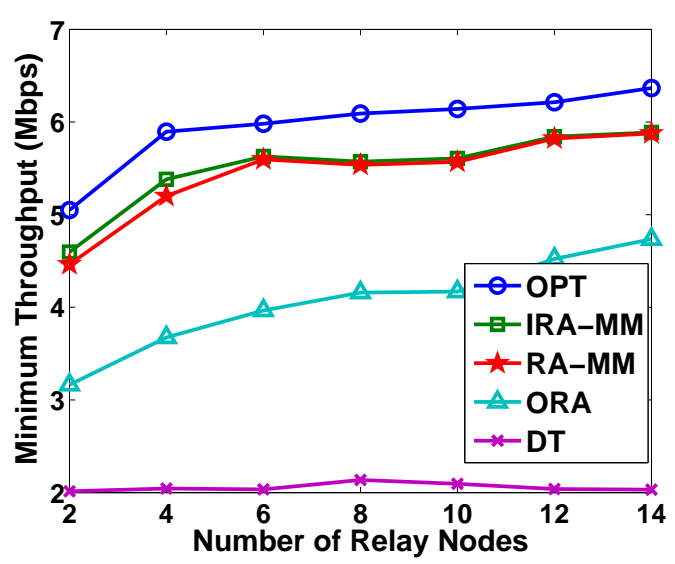

(a) $n_{s}=10$

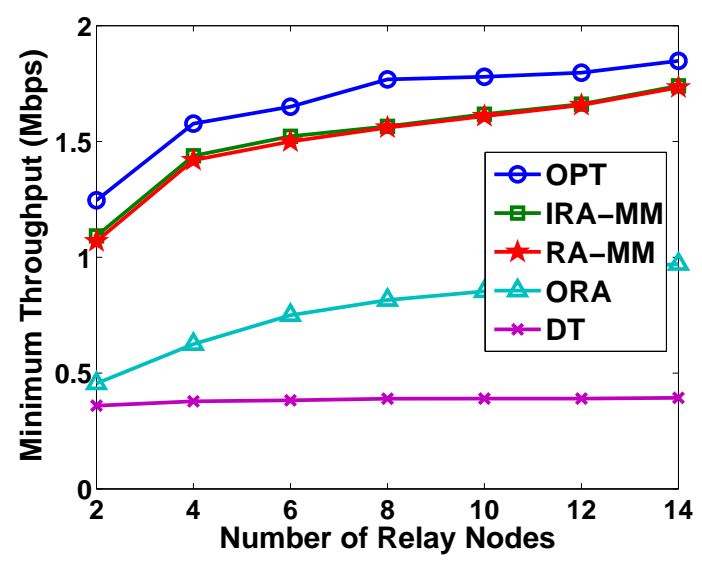

(b) $n_{s}=40$

Fig. 4: Minimum Throughput vs. Number of Relay Nodes

Such a comparison would demonstrate the advantage of the SRA policy in M21 traffic, and our time allocation method. The third one is the OPRA algorithm [16] designed for maxtotal throughout and hence will be compared with our RAMT only. For fair comparison, we also extend it by allocating an equal amount of time to each of the sources sharing the same destination. Such a comparison will also demonstrate the advantage of the SRA policy in M21 traffic. The last benchmark is the upper-bound result for M21-SRA-MMT and M21-SRA-MTT, respectively, denoted by OPT, which can be obtained by solving the linear programs $L P_{1}$ and $\mathrm{LP}_{4}$ in equations (12) and (21), respectively. For performance comparison, we generate 100 random topologies for each simulation, and average the experimental results.

\subsection{Simulation Results for M21-SRA-MMT}

This section mainly observes the minimum throughput in the network for the different algorithms. Fig. 3 mainly illustrates that the minimum throughput decreases when the number of the source nodes increases from 5 to 40 for all algorithms (when the number of relay nodes is fixed). When the number of relay is less, shown in Fig. 3(a), our algorithms can perform much better than the ORA algorithm as there are much more source nodes in the network. For example, the

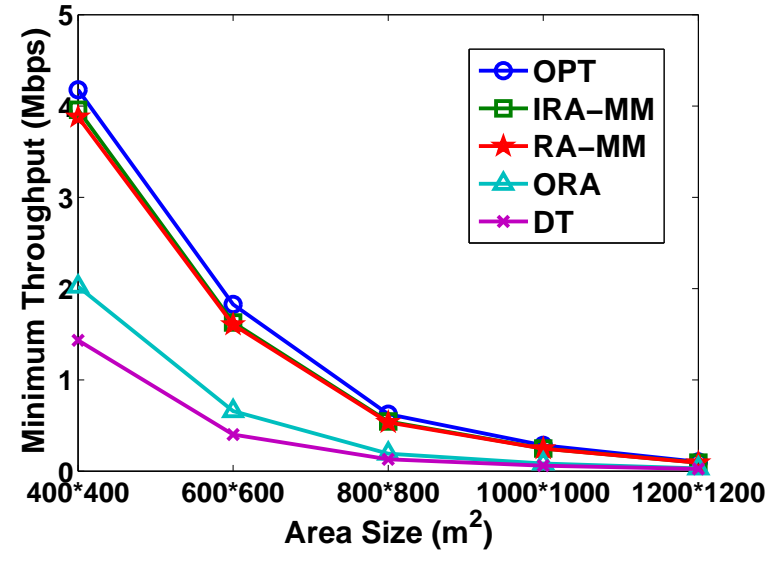

Fig. 5: Minimum Throughput vs. Area Size

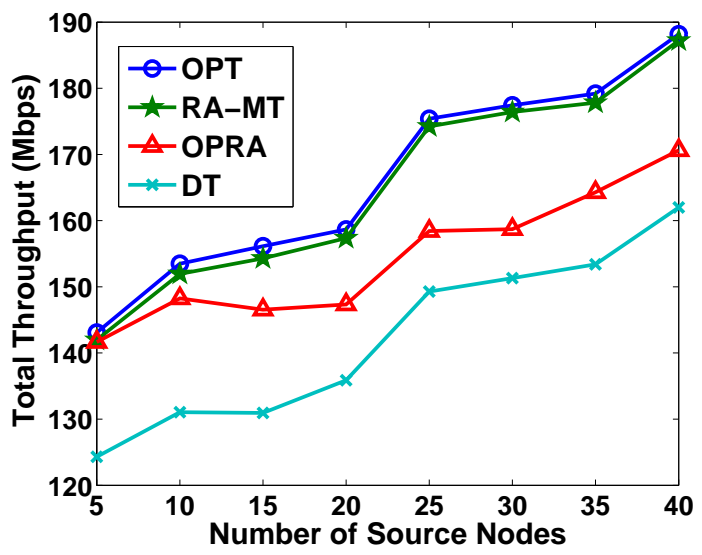

(a) $n_{r}=5$

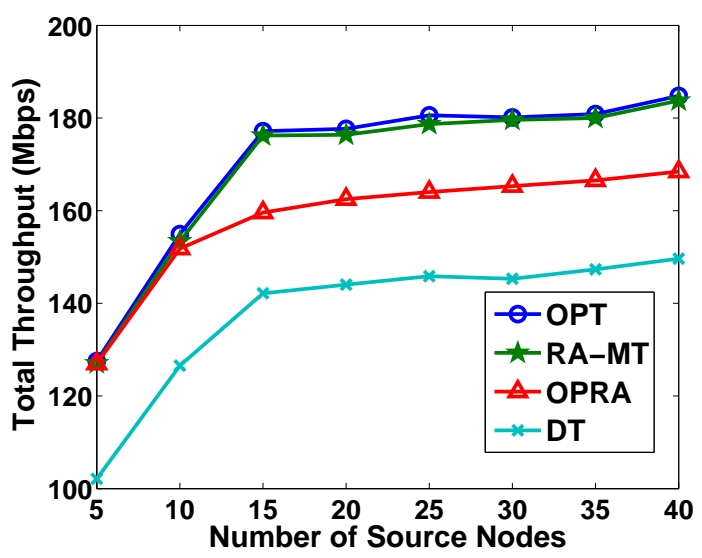

(b) $n_{r}=10$

Fig. 6: Total Throughput vs. Number of Source Nodes

RA-MM and IRA-MM algorithms can improve the minimum throughputs about $34.5 \%$ and $39.1 \%$ compared with ORA when there deploy 5 relay nodes. When there is more relay nodes (e.g., $n_{r}=10$ ), shown in Fig. 3(b), the RA-MM and IRA-MM algorithms improve the minimum throughputs about $23.0 \%$ and $29.1 \%$ compared with the ORA algorithm.

Fig. 4 shows that the minimum throughput increases with the number of the relay nodes (with a fixed number of source 
nodes). From the figures, the minimum throughput is increased when the number of relays is increasing for all algorithms. When the number of source nodes is 10, Fig. 4(a) shows that the IRA-MM algorithm can improve the minimum throughput about $16.6 \%$ compared with ORA. When the number of source nodes is 40 , both algorithms will perform better than the ORA algorithm. Especially, the proposed algorithms can improve the minimum throughput about $54.7 \%$ and $59.6 \%$ compared with ORA on average.

From Fig. 5, the minimum throughput decreases with the area size as expected. This is reasonable since according to Eqs. (1)-(3), the transmission capacity is a decreasing function of the communication distance. Note that, Fig. 5 shows that both our algorithms will perform much better than the ORA algorithm with improvement over $111 \%$ and $117 \%$, respectively.

Also, from three figures, our algorithms can perform much better than the ORA algorithm. For example, when both the numbers of relays and source nodes are small (e.g., both 5), the RA-MM and IRA-MM algorithms can improve the minimum throughput by about $18 \%$ and $20 \%$, respectively compared with ORA, which is the optimal algorithm for the 121-DRAMMT problem. When the number of source nodes is large (e.g., 40) and the number of relays is small (e.g., 5), both algorithms will perform much better than the ORA algorithm with improvement over $110 \%$ and $116 \%$, respectively. When averaged over all the values of the number of sources, and number of relays considered, the RA-MM and IRA-MM algorithms can improve the minimum throughput around $61 \%$ and $66 \%$ compared with ORA.

We observe the standard deviation for different algorithms, and make two conclusions. First, the standard derivation becomes smaller under a denser network (e.g., 30 source nodes) than that under a sparser network (e.g., 10 source nodes) for each algorithm. Second, the standard derivation would increase as the average-case performance is improved.

These results show the significant benefit of adopting the SRA policy (and allocating an appropriate transmission time). In particular, our algorithms can achieve better performance than ORA when there are much more sources than the number of relays. This because, under the DRA policy as in ORA, there are not enough relays available to all the sources using any relays and hence some sources only achieve a low throughput, whereas under the SRA policy as in M21-SRAMMT, many sources can benefit from sharing a small number of relays to increase the minimum throughput in the network.

\subsection{Simulation Results for M21-SRA-MTT}

This section mainly observes the total throughput in the network for the different algorithms. Fig. 6 illustrates the impact of the number of source nodes on the total throughput in the network. From the figure, the total throughput is increased when the number of the source nodes is increasing for all algorithms. When the number of relay is less, shown in Fig. 6(a), our algorithms can perform much better than the OPRA algorithm as there are much more source nodes in the network. In addition, our proposed RA-MT algorithm improves the total

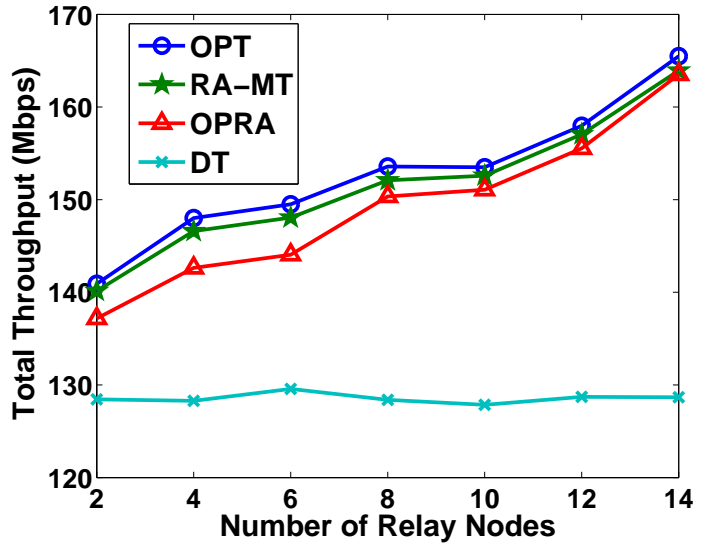

(a) $n_{s}=10$

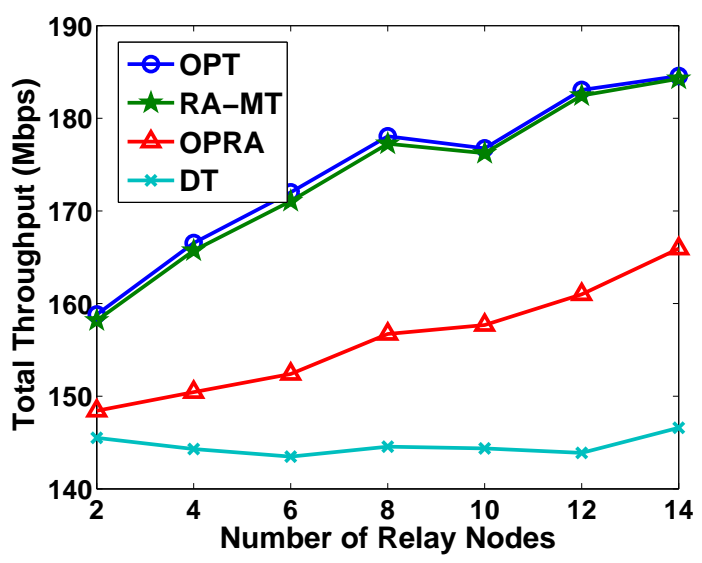

(b) $n_{s}=40$

Fig. 7: Total Throughput vs. Number of Relay Nodes

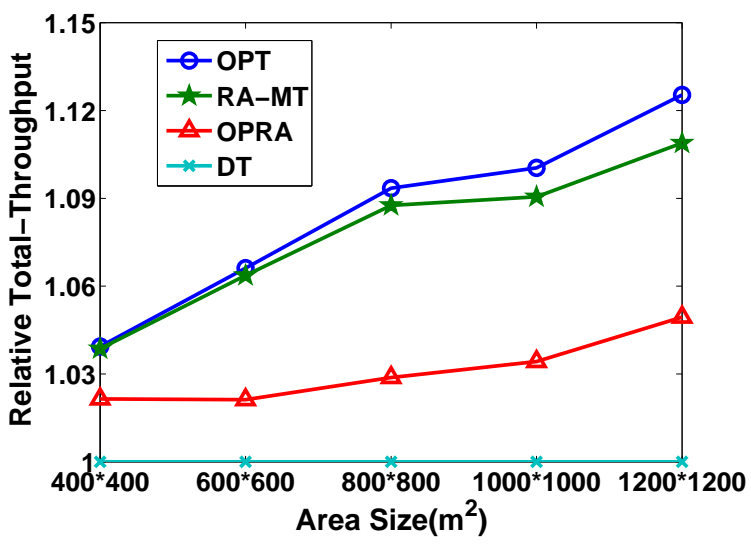

Fig. 8: Total Throughput vs. Area Size

throughput up to $22 \%$ (with $10 \%$ on average) compared with the OPRA algorithm, thanks to the SRA policy.

Fig. 7 illustrates the impact of the number of relay nodes on the total throughput in the network. When the number of source nodes is 10, Fig. 7(a) shows that the RA-MT algorithm can improve the total throughput about $17.9 \%$ compared with OPRA, which is optimal under the DRA policy. When the number of source nodes is 40 , both algorithms will perform 


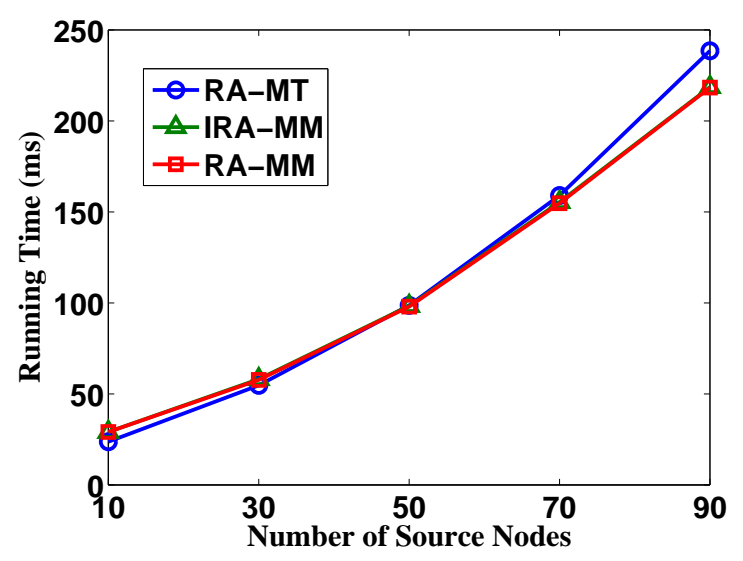

Fig. 9: Running Time vs. Number of Source Nodes

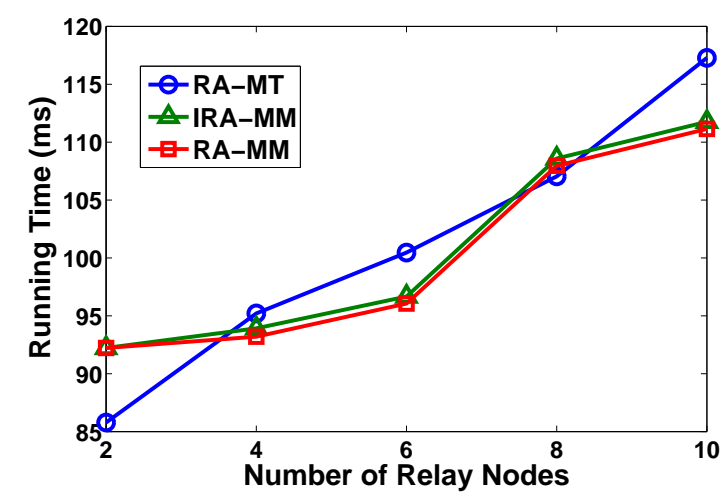

Fig. 10: Running Time vs. Number of Relay Nodes

better than the OPRA algorithm. Especially, the proposed algorithms will increase the total throughput about 20.0\% compared with OPRA on average. Moreover, our proposed algorithm can achieve almost the similar performance compared with OPT, which is an upper-bound for the M21-SRA-MTT problem from the linear program $\mathrm{LP}_{4}$ in equation (21).

We observe the impact of the area size on the total throughput in the network. For ease illustration, Fig. 8 plots the relative total-throughput when the area size varies. In the figure, we assume that the total throughput of the DT scheme is 1 , and compute the total throughput of each other algorithm by compared with that by DT. We can find that our RA-MT algorithm can improve the total throughput much more in a larger area than that in a smaller area.

\subsection{Time Complexity of Our Algorithms}

Under the same simulation setting as described elsewhere in the paper, Figs. 9 and 10 illustrate that the average running times of the three algorithms increase super-linearly (but not exponentially) with the number of source nodes and the number of relay nodes, respectively. Hence, the running times of the three algorithms are quite acceptable in the practical scenarios as expected. For example, when there are 90 source nodes, 5 relay nodes and 5 destination nodes, the running times of the three algorithms are about $220 \mathrm{~ms}$ as shown in Fig. 9 . When when there are 50 source nodes, 10 relay nodes and 5 destination nodes, the running times of the three algorithms are about $115 \mathrm{~ms}$ as shown in Fig. 10.

\section{Other Related Works}

The concept of cooperative communication had been around for many years [21]. This section briefly reviews the related works about relay assignment according to two categories. One is relay assignment for a single transmission pair, the other is focusing on relay assignment among multiple pairs.

For the first category, Nazaroglu et al. [22] studied the Nrelay Gaussian network in which each node was equipped with multiple antennas. They showed that there existed a subnetwork with two relays that could achieve approximately full capacity of the network in some situations. As mentioned earlier, Zhao et al. [11] proved that it was enough to choose the best relay node for a transmission pair to achieve the spatial diversity. Therefore, the previous works mostly assumed that each transmission pair would use at most one relay node for cooperative communication. For example, Bletsas et al. [12] designed the approach to select an optimal relay node for a single source-destination pair. In [23], the source node decided whether to employ the relay in forwarding the information or not, depending on the instantaneous channel gains. In [24], the source node decided when to cooperate by taking the ratio between the $s-d$ channel gain and the optimal relay's metric, and selected the optimal relay for cooperative transmission. The studies in [5] [11] [23] [24] are limited to one $s-d$ pair, and cannot be easily extended to a network scene where multiple $s-d$ transmission pairs compete for a relay set, which is the focus of this paper.

With respect to the second category, there are some studies on relay assignment among multiple transmission pairs with different destination nodes to satisfy the different requirements such as the works in [1], [15] and [16] mentioned earlier. Following the work [1], Zhang et al. [25] proposed a relay assignment solution to minimize the network interference by the bipartite matching. Xu et al. [26] designed the efficient relay assignment algorithms to optimize the proportional fairness and lexicographical max-min fairness for wireless networks. In [27], $\mathrm{Ng}$ and $\mathrm{Yu}$ considered the relay selection, cooperative communication and resource allocation for utility maximization in a cellular network. However, the algorithm could not be finished in the polynomial time [1]. Cai et al. [15] studied the joint relay selection and power allocation problem for the $A F$ mode. They considered the different network cases with only one-source and multiple-source. However, the proposed algorithm could guarantee the optimal or approximate performance. $\mathrm{Xu}$ et al. [28] studied the relay assignment problem to minimize the total power consumption while the required bandwidth of each transmission pair was satisfied. Then, they proposed a matching-based algorithm to solve this problem. The work in [29] formulated the problem of cooperative relay selection in a cognitive radio network as a stopping problem, and derived the optimal rule for relay selection. Cao et al. [30] proposed two auction schemes for relay assignment in wireless cooperative networks. The work in [31] studied the shared relay assignment for multiple independent transmission pairs 
so as to increase the minimum throughput. Sharma et al. [32] studied the joint grouping and relay node selection problem for network-coded cooperative communications. These studies all assume the multiple transmission pairs with different destinations in networks. However, multiple sources may associate with one destination, such as in the WLAN, which is more general than the model in the above researches. Moreover, the SRA policy is also forbidden in almost all these works. Finally, the work in [33]-[35] studied "relay" placement for the M21 traffic in wireless sensor networks while such relays do not participate in cooperative communications and hence, differ from ours.

\section{CONCLUSION}

In this paper, we have studied two new shared relay (node) assignment (SRA) problems for many-to-one (M21) traffic in the context of cooperative wireless communications, namely M21-SRA-MMT and M21-SRA-MTT, in order to maximize the minimum and total throughputs. We have designed two rounding-based algorithms (RA-MM and RA-MT), whose worst-case performance is guaranteed by their approximate factors of 5.828 and 3, to maximize the minimum and total throughputs respectively. We have also proposed the variation of RA-MM to improve the average-case performance while guaranteeing the worst-case performance. Our simulations show that the proposed algorithm for M21-SRA-MMT can achieve about $66 \%$ improvement in the minimum throughout compared with ORA, while the proposed algorithm for M21SRA-MTT can achieve close-to-optimal performance.

Note that, our solutions apply to the cases where there are both one-to-one and many-to-one traffics. In addition, we expect our work to inspire a lot of follow-up researches since the notion of SRA can be generalized. More specifically, let us call a set of source nodes that communicate with the same destination node a group. There can be two main variations for each of the M21-SRA-MMT and M21-SRA-MTT problems studied in this paper. One variation is that the DRA policy is applied to intra-group communications, while SRA is applied to inter-group communications. That is, only the source nodes from the different groups can possibly share the same relay node. The other variation is that the DRA policy is applied to inter-group, while SRA is applied to intra-group. As the above two variations are special cases of the M21-SRA-MMT and M21-SRA-MTT problems, we can modify the proposed RA-MM and RA-MT algorithms a little so as to satisfy the constraints of relay assignment. However, these algorithms cannot guarantee the (worst-case) approximation performance. As a future work, we will design other algorithms with guaranteed (worst-case) performance for these and other relay assignment problems. Moreover, another future work is, by starting from the proposed three algorithms, to design the efficient shared relay assignment mechanisms that can adapt to a dynamic and mobile wireless environment.

\section{APPENDIX A Proof of Lemma 1}

Proof: Clearly, the problem is in NP, since we can verify in polynomial time if a candidate solution can accomplish the shared relay assignment and achieve the minimum throughput constraint.

We prove the NP-hardness by showing that the association control problem [14] is a special case of the M21-SRAMMT problem. In the WLAN network, the association control problem is to associate each terminal with one AP so as to maximize the minimum throughput of all the terminals. We consider a special case of M21-SRA-MMT, in which each destination is only connected with one source. This is just the 121-SRA-MMT problem. Next, we will show that the association control problem and the 121-SRA-MMT problem are equivalent. For the association control problem, there are $n$ terminals, denoted by $\left\{v_{1}, \ldots, v_{n}\right\}$, and $m$ APs, denoted by $\left\{u_{1}, \ldots, u_{m}\right\}$. Moreover, the transmission capacity between terminal $v_{i}$ and $\mathrm{AP} u_{i}$ is denoted by $C_{i j}$. Then, we will transform this instant into one instant of the 121-SRAMMT problem as follows: the network consists of $m$ relays, denoted by $\left\{r_{1}, \ldots, r_{m}\right\}$ and $n$ transmission pairs, denoted by $\left\{\Gamma_{1}, \ldots, \Gamma_{n}\right\}$. The cooperative transmission capacity that the transmission pair $\Gamma_{i}$ uses a relay $r_{j}$ is denoted by $C_{i j}$. It should be noted that APs and terminals in the association control problem correspond to relays and transmission pairs in the 121-SRA-MMT problem, respectively. Then, the association control problem is equivalent to the 121-SRA-MMT problem. In other words, a solution to the 121-SRA-MMT problem can be transformed into another solution to the association control problem, and vice versa. As a result, the association control problem is a special case of the M21-SRA-MMT problem. Thus, M21-SRA-MMT is an NP-Hard problem too.

\section{ACKNOWLEDGEMENT}

Some preliminary results of this paper were published in the Proceedings of IEEE/ACM IWQoS 2014 [36]. This paper is supported by the National Science Foundation of China under Grant No. U1301256, 61170058, 61272133, 61472383 and 51274202 , Special Project on IoT of China NDRC (20122766), China Postdoctoral Science Foundation project under Grant No. 2012T50547, Research Fund for the Doctoral Program of Higher Education of China No. 20123402110019, the NSF of Jiangsu Province Grant No. BK2012632, and Suzhou Industry Fund Grant No. SYG201302.

\section{REFERENCES}

[1] Yi Shi, Sushant Sharma, Y Thomas Hou, and Sastry Kompella. Optimal relay assignment for cooperative communications. In Proceedings of the 9th ACM international symposium on Mobile ad hoc networking and computing, pages 3-12. ACM, 2008.

[2] Shuguang Cui, Andrea J Goldsmith, and Ahmad Bahai. Energyefficiency of mimo and cooperative mimo techniques in sensor networks. Selected Areas in Communications, IEEE Journal on, 22(6):1089-1098, 2004.

[3] J Nicholas Laneman, David NC Tse, and Gregory W Wornell. Cooperative diversity in wireless networks: Efficient protocols and outage behavior. Information Theory, IEEE Transactions on, 50(12):3062-3080, 2004.

[4] Andrew Sendonaris, Elza Erkip, and Behnaam Aazhang. User cooperation diversity. part i. system description. Communications, IEEE Transactions on, 51(11):1927-1938, 2003.

[5] Dejun Yang, Xi Fang, and Guoliang Xue. Truthful auction for cooperative communications. In Proceedings of the Twelfth ACM International Symposium on Mobile Ad Hoc Networking and Computing, page 9. ACM, 2011. 
[6] Lei Ding, Tommaso Melodia, Stella N Batalama, and John D Matyjas. Distributed routing, relay selection, and spectrum allocation in cognitive and cooperative ad hoc networks. In Sensor Mesh and Ad Hoc Communications and Networks (SECON), 2010 7th Annual IEEE Communications Society Conference on, pages 1-9. IEEE, 2010.

[7] Jianwei Huang, Zhu Han, Mung Chiang, and H Vincent Poor. Auctionbased resource allocation for cooperative communications. Selected Areas in Communications, IEEE Journal on, 26(7):1226-1237, 2008.

[8] Goeckel D.L. Laneman J.N. Scaglione, A. Cooperative communications in mobile ad hoc networks. IEEE Signal Processing Magazine, 6(3): 1053-1064, 2006.

[9] Yang S Cardei M, Wu J. Topology control in ad hoc wireless networks using cooperative communication. Mobile Computing, IEEE Transactions on, 5(6):711-724, 2006.

[10] Liang-Liang Xie Xuemin Shen Tapolcai Bin Lin, Pin-Han Ho. Optimal relay station placement in broadband wireless access networks. Mobile Computing, IEEE Transactions on, 9(2):259-269, 2010.

[11] Yi Zhao, Raviraj Adve, and Teng Joon Lim. Improving amplify-andforward relay networks: optimal power allocation versus selection. In Information Theory, 2006 IEEE International Symposium on, pages 1234-1238. IEEE, 2006.

[12] Aggelos Bletsas, Ashish Khisti, David P Reed, and Andrew Lippman. A simple cooperative diversity method based on network path selection. Selected Areas in Communications, IEEE Journal on, 24(3):659-672, 2006.

[13] Jun Cai, Xuemin Shen, Jon W Mark, and Attahiru S Alfa. Semidistributed user relaying algorithm for amplify-and-forward wireless relay networks. Wireless Communications, IEEE Transactions on, 7(4):1348-1357, 2008.

[14] Yigal Bejerano, Seung-Jae Han, and Li Erran Li. Fairness and load balancing in wireless lans using association control. In Proceedings of the 10th annual international conference on Mobile computing and networking, pages 315-329. ACM, 2004.

[15] Sushant Sharma, Yi Shi, Y Thomas Hou, and Sastry Kompella. An optimal algorithm for relay node assignment in cooperative ad hoc networks. IEEE/ACM Transactions on Networking (TON), 19(3):879892, 2011

[16] Dejun Yang, Xi Fang, and Guoliang Xue. Opra: Optimal relay assignment for capacity maximization in cooperative networks. In Communications (ICC), 2011 IEEE International Conference on, pages 1-6. IEEE, 2011.

[17] James Munkres. Algorithms for the assignment and transportation problems. Journal of the Society for Industrial \& Applied Mathematics, 5(1):32-38, 1957.

[18] Zijian Mo, Weifeng Su, Stella Batalama, and J Matyjas. Cooperative communication protocol designs based on optimum power and time allocation. 2014.

[19] David B Shmoys and Éva Tardos. An approximation algorithm for the generalized assignment problem. Mathematical Programming, 62(13):461-474, 1993.

[20] Chandra Chekuri and Sanjeev Khanna. A ptas for the multiple knapsack problem. In Proceedings of the Eleventh Annual ACM-SIAM Symposium on Discrete Algorithms, SODA '00, pages 213-222, Philadelphia, PA, USA, 2000. Society for Industrial and Applied Mathematics.

[21] T Cover and A EL Gamal. Capacity theorems for the relay channel. Information Theory, IEEE Transactions on, 25(5):572-584, 1979.

[22] Caner Nazaroglu, Javad B Ebrahimi, A Ozgur, and Christina Fragouli. Network simplification: the gaussian diamond network with multiple antennas. In Information Theory Proceedings (ISIT), 2011 IEEE International Symposium on, pages 79-83. IEEE, 2011.

[23] Ahmed S Ibrahim, Ahmed K Sadek, Weifeng Su, and KJ Ray Liu. Cooperative communications with partial channel state information: when to cooperate? In Global Telecommunications Conference, 2005. GLOBECOM'05. IEEE, volume 5, pages 5-pp. IEEE, 2005.

[24] Ahmed S Ibrahim, Ahmed K Sadek, Weifeng Su, and KJ Ray Liu. Cooperative communications with relay-selection: when to cooperate and whom to cooperate with? Wireless Communications, IEEE Transactions on, 7(7):2814-2827, 2008.

[25] Peng Zhang, Zhengguang Xu, Furong Wang, Xu Xie, and Lai Tu. A relay assignment algorithm with interference mitigation for cooperative communication. In Wireless Communications and Networking Conference, 2009. WCNC 2009. IEEE, pages 1-6. IEEE, 2009.

[26] Hongli Xu, Liusheng Huang, Gang Liu, Yindong Zhang, and He Huang. Optimal relay assignment for fairness in wireless cooperative networks. International Journal of Ad Hoc and Ubiquitous Computing, 9(1):42-53, 2012.
[27] Truman Chiu-Yam Ng and Wei Yu. Joint optimization of relay strategies and resource allocations in cooperative cellular networks. Selected Areas in Communications, IEEE Journal on, 25(2):328-339, 2007.

[28] Hongli Xu, Liusheng Huang, Gang Wang, Tao Xu, and Gang Liu. Joint relay assignment and power allocation for cooperative communications. Wireless Networks, 16(8):2209-2219, 2010.

[29] Tao Jing, Shixiang Zhu, Hongjuan Li, Xiuzhen Cheng, and Yan Huo. Cooperative relay selection in cognitive radio networks. In INFOCOM, 2013 Proceedings IEEE, pages 175-179. IEEE, 2013.

[30] Bin Cao, Gang Feng, Yun Li, and Mahmoud Daneshmand. Auctionbased relay assignment in cooperative communications. In Global Communications Conference (GLOBECOM), 2014 IEEE, pages 44964501. IEEE, 2014.

[31] Hongli Xu, Liusheng Huang, Hou Deng, Chunming Qiao, and Yude Lin. Fairness-aware shared relay assignment for cooperative communications. In Communications (ICC), 2014 IEEE International Conference on, pages 5700-5705. IEEE, 2014.

[32] Sushant Sharma, Yi Shi, Y Thomas Hou, Hanif D Sherali, and Sastry Kompella. Joint optimization of session grouping and relay node selection for network-coded cooperative communications. Mobile Computing, IEEE Transactions on, 13(9):2028-2041, 2014.

[33] Feng Wang, Dan Wang, and Jiangchuan Liu. Traffic-aware relay node deployment for data collection in wireless sensor networks. In Sensor, Mesh and Ad Hoc Communications and Networks, 2009. SECON'09. 6th Annual IEEE Communications Society Conference on, pages 1-9. IEEE, 2009.

[34] Yuanteng Pei and Matt W Mutka. Joint bandwidth-aware relay placement and routing in heterogeneous wireless networks. In Parallel and Distributed Systems (ICPADS), 2011 IEEE 17th International Conference on, pages 420-427. IEEE, 2011.

[35] Rajeev Paulus, Gurmit Singh, and Rajiv Tripathi. Energy efficient data transmission through relay nodes in wireless sensor networks. International Journal of Network Security (2152-5064), 3(1), 2012.

[36] Hongli Xu, Liusheng Huang, Chunming Qiao, Xinglong Wang, and Yu-e Sun. Shared relay assignment (sra) for many-to-one traffic in cooperative wireless transmissions. IWQoS, 2014.

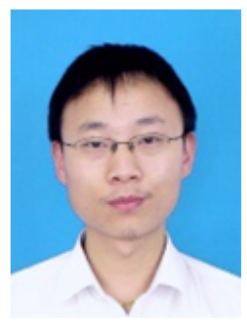

Hongli $\mathrm{Xu}$ received his $\mathrm{Ph}$. D degree in Computer Science from the University ofalg:2 Science and Technology of China in 2007. Currently, he is an assistant research fellow in the School of Computer Science and Technology at the University of Science and Technology of China. His main research interest is cooperative communication, vehicular ad hoc network and software defined networks.

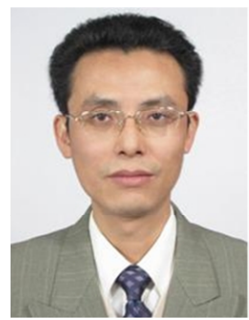

Liusheng Huang received the M.S. degree in computer science from University of Science and Technology of China in 1988. He is currently a professor and Ph.D. supervisor of the School of Computer Science and Technology at the University of Science and Technology of China. $\mathrm{He}$ has published 6 books and more than 200 papers. His research interests are in the areas of internet of thing, vehicular ad hoc network, information security, and distributed computing. 


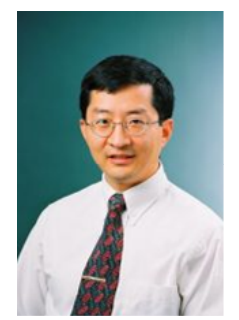

Chunming Qiao directs the Lab for Advanced Network Design, Analysis, and Research (LANDER) at SUNY Buffalo. He is a recipient of many awards including the SUNY Chancellors Award for Excellence in Scholarship and Creativity. He has published many highly cited papers with an h-index of over 50 (according to Google Scholar). He has seven US patents. He pioneered research on Optical Internet in 1997, and one of his papers has been cited by more than 2,000 times. He also pioneered the work on integrated cellular and ad hoc relaying systems (iCAR) in 1999, which is recognized as the harbinger for today's push towards the convergence between heterogeneous wireless technologies, and has been featured in BusinessWeek and Wireless Europe, as well as at the websites of New Scientists and CBC. These works have inspired a lot of followup works around the world and attracted funding from a dozen of major IT and telecommunications companies including Alcatel, Cisco, Google, NEC labs, Nokia, and Telcordia. Dr. Qiao has given a dozen of keynotes, and numerous invited talks on the above research topics. $\mathrm{He}$ has chaired and co-chaired a dozen of international conferences and workshops. He was an editor of several leading IEEE journals, and chaired a number of technical committees. He was elected to IEEE Fellow for his contributions to optical and wireless network architectures and protocols.

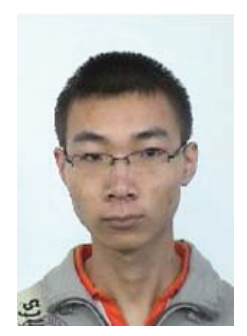

Xinglong Wang is currently a doctor-candidate in Computer Science at the University of Science and Technology of China. He will receive the doctor degree in 2017. His main research interest is VMIMO communication and wireless sensor networks.

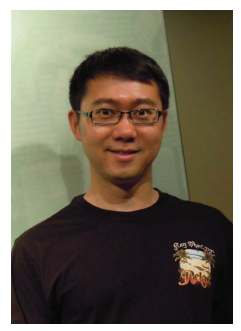

Shan Lin is an assistant professor of the Electrical and Computer Engineering Department at Stony Brook University. He received his $\mathrm{PhD}$ in computer science working under the direction of Professor John A. Stankovic at the University of Virginia in 2010. His PhD dissertation is on Taming Networking Challenges with Feedback Control. His research is in the area of networked systems, with an emphasis on feedback control based design for cyber physical systems and sensor systems. He works on wireless network protocols, interoperable medical devices, smart transportation systems, and intelligent sensing systems.

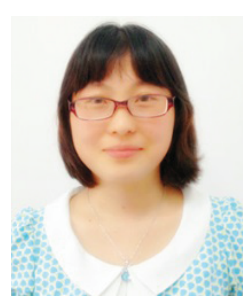

Yu-e Sun is a lecturer of Urban Rail Transportation Department, Soochow University, China. She has received her Ph.D degree in Shenyang Institute of Computing Technology from Chinese Academy of Science. Her current research interests span privacy preserving in spectrum auction, wireless sensor networks, algorithm design and analysis for wireless networks, and network security. She is a member of ACM. 